\title{
Measurements of the differential jet cross section as a function of the jet mass in dijet events from proton-proton collisions at $\sqrt{s}=13 \mathrm{TeV}$
}

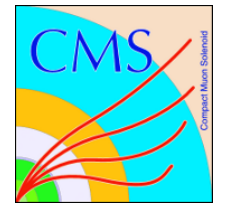

\section{The CMS collaboration}

E-mail: cms-publication-committee-chair@cern.ch

ABSTRACT: Measurements of the differential jet cross section are presented as a function of the jet mass in dijet events, in bins of jet transverse momentum, with and without a jet grooming algorithm. The data have been recorded by the CMS Collaboration in protonproton collisions at the LHC at a center-of-mass energy of $13 \mathrm{TeV}$ and correspond to an integrated luminosity of $2.3 \mathrm{fb}^{-1}$. The absolute cross sections show slightly different jet transverse momentum spectra in data and Monte Carlo event generators for the settings used. Removing this transverse momentum dependence, the normalized cross section for ungroomed jets is consistent with the prediction from Monte Carlo event generators for masses below $30 \%$ of the transverse momentum. The normalized cross section for groomed jets is measured with higher precision than the ungroomed cross section. Semi-analytical calculations of the jet mass beyond leading logarithmic accuracy are compared to data, as well as predictions at leading order and next-to-leading order, which include parton showering and hadronization. Overall, in the normalized cross section, the theoretical predictions agree with the measured cross sections within the uncertainties for masses from 10 to $30 \%$ of the jet transverse momentum.

KEYwORDS: Hadron-Hadron scattering (experiments), Jet physics

ARXiv EPRINT: 1807.05974 


\section{Contents}

1 Introduction 1

2 Theoretical predictions 3

3 Experimental setup $\quad 4$

4 Analysis strategy $\quad 7$

5 Results 12

$\begin{array}{llr}6 & \text { Summary } & 16\end{array}$

$\begin{array}{ll}\text { The CMS collaboration } & 21\end{array}$

\section{Introduction}

The understanding of the evolution of jets in quantum chromodynamics (QCD) is important to the understanding of physics at hadron colliders. Jets are involved in nearly every final state in proton-proton (pp) collisions. In the past, most predictions of jet properties did not focus on the jet mass $m$, defined as the mass of the sum of the four-momenta of the constituents inside the jet. However, the jet mass is the simplest observable that is nonzero only beyond leading order (LO) in perturbation theory for dijet production. Thus, it is more sensitive to the internal structure and evolution of jets than the jet transverse momentum $p_{\mathrm{T}}$. The evolution of jets is theoretically described by parton showering as reviewed in refs. [1, 2]. Experimental measurements of the jet mass provide an important test of these theoretical concepts.

Jet mass has been used extensively to investigate signals of physics beyond the standard model (BSM) that are reviewed in refs. [3-5]. In particular, the jet mass generated by QCD radiation is typically lower than that generated by heavy hadronically decaying standard model (SM) particles that have 2- and 3-prong decays, such as top quarks, W bosons, Z bosons, or Higgs $(\mathrm{H})$ bosons. This observation can be exploited to identify BSM particles that decay into highly Lorentz-boosted SM particles (boosted objects).

The jet mass generated by QCD arises from the radiation of gluons from the higherenergy (hard) portion of the jet. The probability that a gluon is radiated increases as its energy or angle of emission decreases (soft or collinear radiation, respectively). Hence, the probability of having $m=0$ vanishes since many soft and/or collinear gluons will be radiated. Above $m / p_{\mathrm{T}} \approx 0.1$, there is an overall decrease in the jet mass spectrum as the dominant contributions come from gluon emissions with high energy or at large angles. The distribution terminates around $m / p_{\mathrm{T}} \approx 0.3$ for jets with $R=0.8$ (where $R$ is the distance parameter of the algorithm). At that point, the jets become wide enough to start 
splitting into two separate jets (the splitting threshold). The appearance of a large peak at low mass is described in ref. [6] as a "Sudakov peak". The jet mass is sensitive to soft QCD effects and to additional pp interactions in the same bunch crossing (pileup) [7]. There is further sensitivity in hadron colliders to correlations among soft gluon emissions from underlying event interactions, and wide-angle emissions among different jets. While the impacts on global event-wide observables (such as the event thrust) have been understood for some time [8-11], the impact on nonglobal jet observables, such as mass, are difficult to calculate. The technical difficulty arises from the treatment of nonglobal logarithms (NGLs) in the perturbative next-to-leading logarithmic (NLL) order calculations of the jet mass that arise from radiation that exits the jet re-radiating soft gluons back into the jet. It is currently unknown how to fully account for these NGLs in the perturbative integrals.

The hard part of the jet can be separated from the soft parts using jet-grooming techniques [3-5]. These techniques also reduce the effects of pileup, which is not correlated with the hard part of the jet. The earliest grooming techniques were unable to completely remove the soft parts of a jet systematically. More recently, major improvements have been made to separate the hard and soft parts of the jet without contamination from NGLs [6, 8, 12-15]. The "mass drop" (MD) procedure [16] has been modified into such a theoretically controlled jet-grooming algorithm [6]. Its generalization, the "soft drop" (SD) algorithm [12], has similar properties. By applying the SD algorithm, soft parts of a jet are removed, resulting in a dramatically reduced Sudakov peak in the jet mass distribution. This also systematically removes the effects that cause NGLs in the calculation, allowing for direct comparisons of the semi-analytical NLL resummation calculations (in particular, from the authors of refs. [17] and [18]) to Monte Carlo (MC) simulated predictions and data. We therefore apply the SD procedure to groom the jets in this paper. A similar analysis has been published by the ATLAS Collaboration in ref. [19]. Understanding the behavior of the jet mass and the grooming techniques from first principles is important to further develop boosted object identification algorithms, to gain confidence in the tools should they uncover BSM physics, and to check that parton showering algorithms are correct.

In this paper, we present a measurement of the differential jet production cross section as a function of the jet mass in bins of $p_{\mathrm{T}}$ from dijet events, before and after the jets are groomed with the SD procedure (for our choice of parameters the SD and MD procedures are identical). Comparing the production cross section with respect to the groomed $\left(m_{\mathrm{g}}\right)$ and ungroomed $\left(m_{\mathrm{u}}\right)$ jet mass separately allows us to gain insight into both the hard and soft physics.

This analysis is similar to that of ref. [7], except we correct both the jet $p_{\mathrm{T}}$ and $m$ for detector effects, and the SD algorithm is used. We present the double-differential production cross section of dijet events with respect to the jet $p_{\mathrm{T}}$ and jet mass $m$ (where $m=m_{\mathrm{u}}$ or $\left.m_{\mathrm{g}}\right)$ :

$$
\frac{\mathrm{d}^{2} \sigma}{\mathrm{d} p_{\mathrm{T}} \mathrm{d} m}\left(\mathrm{pb} / \mathrm{GeV}^{2}\right)
$$

In addition, we define a normalized distribution:

$$
\frac{1}{\mathrm{~d} \sigma / \mathrm{d} p_{\mathrm{T}}} \frac{\mathrm{d}^{2} \sigma}{\mathrm{d} p_{\mathrm{T}} \mathrm{d} m}(1 / \mathrm{GeV})
$$


In both cases, the two jets in the dijet system are included in the distributions. We have used only the $2.3 \mathrm{fb}^{-1}$ dataset instead of all the available luminosity, because we present the ungroomed cross section, which is systematically limited by the pileup uncertainty in higher luminosity data samples.

\section{Theoretical predictions}

There are five theoretical calculations used in this study: three $\mathrm{MC}$ event generator predictions, and two predictions using analytic resummation merged with fixed order $\mathrm{MC}$ simulations.

The three MC event generators used in this study are PYTHIA8, HERWIG++, and POWHEG. Version 8.212 of PYTHIA8 [20] is used with underlying event tune CUETP8M1 [21] and NNPDF 3.0 parton distribution functions (PDFs) NNPDF30_lo_as_0130 [22]. The PDF4LHC15_NLO_100 meta-PDF set is used for uncertainty estimation, along with the PYTHIA8 generator. We use the LHAPDF [23] software package for this purpose, following the recommendations of ref. [24]. The HERWIG ++ version 2.7.0 generator [25] is used with tune CUETHS1 [21]. The HERWIG++ simulation sample is used for systematic uncertainty estimation and for generator-level comparisons, as it has a physics model that is different from PYTHIA 8 in various aspects like parton showering, hadronisation, multiple-parton interactions, and the set of model parameters tuned to data. Finally, the POWHEG 2.0 [26-29] generator interfaced with PYTHIA8 is used as a next-to-leading order (NLO) perturbative dijet event generator, supplemented with parton showering and multiparton interactions. The same underlying event tune CUETP8M1 [21] is used.

Two semi-analytical resummation-based calculations merged with fixed-order MC simulation are also used. The authors of ref. [17] have provided a prediction of the groomed jet mass distribution for dijet events at LO using MADGRAPH v2.4.2 [30] and NNPDF 3.0 LO PDFs [22], resummed at NLL accuracy using soft collinear effective theory. Nonperturbative effects are included as an uncertainty in their prediction. The authors of ref. [18] have provided a prediction of the groomed jet mass distribution for dijet events at NLO using NLOJET $++[31]$ and CT14NLO PDFs [32] with NLL resummation using perturbative QCD. They include an estimate of the nonperturbative effects directly, using PYTHIA8.

The semi-analytical predictions are expected to be accurate for intermediate masses $0.1<m / p_{\mathrm{T}}<0.3$, since at low mass nonperturbative effects are large. Moreover, at high mass the resummed predictions are matched to fixed-order predictions at LO and NLO, which cannot account for more than one or two additional gluons, respectively, as would occur within genuine jets. For a single gluon emission, there is a kinematic upper limit at $m=p_{\mathrm{T}} R / 2$ (where $R=\sqrt{(\Delta \eta)^{2}+(\Delta \phi)^{2}}$ is the distance parameter for the jet clustering), from the relativistic kinematics of a $1 \rightarrow 2$ decay. However, in the all-orders limit, the approximate kinematic limit is closer to $m=p_{\mathrm{T}} R / \sqrt{2}$. Consider a particle of energy $E$ and mass $m$ decaying to two massless particles, each with an energy $E / 2$ and separated by an angle $\theta$. The mass must satisfy $m^{2}<E^{2}(1-\cos \theta) / 2$. In the small-angle limit, this would be $m^{2}<E^{2} \theta^{2} / 4$, or $m<E \theta / 2$. With more particles, the stochastic nature of the shower increases this cutoff point. Thus, a single emission $(1 \rightarrow 2)$ decay will have a 
lower approximate kinematic limit than an all-orders $(1 \rightarrow$ many) decay. Reference [18] calculates these thresholds to be $m / E \approx 0.28$ at LO and $m / E \approx 0.45$ at NLO.

\section{$3 \quad$ Experimental setup}

The central feature of the CMS detector [33] is a superconducting solenoid of $6 \mathrm{~m}$ internal diameter, providing a magnetic field of $3.8 \mathrm{~T}$. Within the superconducting solenoid volume are a silicon pixel and strip tracker, a lead tungstate crystal electromagnetic calorimeter (ECAL), and a brass and scintillator hadron calorimeter (HCAL), each split in the barrel and two endcap sections. In the region $|\eta|<1.74$, the HCAL cells have widths of 0.087 in pseudorapidity $(\eta)$ and 0.087 radians in azimuth $(\phi)$. In the $\eta$ - $\phi$ plane, and for $|\eta|<1.48$, the HCAL cells map onto $5 \times 5$ ECAL crystal arrays to form calorimeter towers projecting radially outwards from close to the nominal interaction point. At larger values of $|\eta|$, the size of the towers increases and the matching ECAL arrays contain fewer crystals. Muons are measured in gas-ionization detectors embedded in the steel flux-return yoke outside the solenoid. Extensive forward calorimetry complements the coverage provided by the barrel and endcap detectors. Electron momenta are estimated by combining the energy measurement in the ECAL with the momentum measurement in the tracker. In this analysis, the jet rapidity $(y)$ is used in the computation of jet quantities. A more detailed description of the CMS detector, together with a definition of the coordinate system used and the relevant kinematic variables, can be found in ref. [33].

Events of interest are selected using a two-tiered trigger system [34]. The first level (L1), composed of custom hardware processors, uses information from the calorimeters and muon detectors to select events at a rate of around $100 \mathrm{kHz}$ with a latency of less than $4 \mu \mathrm{s}$. The second level, known as the high-level trigger (HLT), consists of a farm of processors running a version of the full event reconstruction software optimized for fast processing, and reduces the event rate to around $1 \mathrm{kHz}$ before data storage.

The event reconstruction is based on the CMS particle-flow (PF) algorithm [35], which includes information from all subdetectors, including charged-particle tracks from the tracking system, energy deposits in the ECAL and HCAL, and tracks reconstructed in the muon chambers. Particles in an event are reconstructed and identified as electrons, muons, photons, charged hadrons, or neutral hadrons. Charged hadrons associated with pileup vertices are removed from consideration. This method is referred to as "charged-hadron subtraction" (CHS) [36]. In our dataset, the average number of pileup interactions per beam crossing is 14 .

Jets are clustered using the anti- $k_{\mathrm{T}}$ algorithm [37] with the FASTJET 3.0 software package [38], with a distance parameter of $R=0.8$ (AK8 jets). This distance parameter is chosen to coincide with the majority of CMS BSM searches using jet substructure techniques. To reconstruct the groomed jets, the constituents of the AK8 jets are reclustered using the Cambridge-Aachen algorithm $[39,40]$ with the same characteristic radius (CA8) to retain substructure information. The hierarchical sequence of clustering is then reversed ("declustering"). The jet grooming algorithm used is the SD algorithm [12]. For each 
declustering step, the "soft-drop condition" is applied:

$$
\frac{\min \left(p_{\mathrm{T}, i}, p_{\mathrm{T}, j}\right)}{\left(p_{\mathrm{T}, i}+p_{\mathrm{T}, j}\right)}>z_{\mathrm{cut}}\left(\frac{\Delta R_{i j}}{R_{0}}\right)^{\beta},
$$

where $p_{\mathrm{T}, i}$ and $p_{\mathrm{T}, j}$ are the transverse momenta of the $i$ th and $j$ th particle at each declustering step, $\Delta R_{i j}$ is the distance between them, $z_{\text {cut }}$ corresponds to the threshold to separate the "soft" and "hard" parts of the jet, and $\beta$ is an angularity exponent. If any declustering step does not satisfy the soft-drop condition, the softer constituent is removed from consideration by the jet algorithm. We choose $\beta=0, z_{\text {cut }}=0.1$ and hence $R_{0}$ is no longer a relevant parameter. For this choice of parameters, the SD algorithm is identical to the variant of the MD algorithm described in ref. [6]. The choice of $\beta=0$ was made for simplicity to agree with most other CMS analyses, whereas other values of $\beta$ will be addressed in future work. The measurement is not very sensitive to the value of $z_{\text {cut }}$, and is chosen to be consistent with ref. [6] and other CMS analyses.

For the reconstructed jets, all PF candidates that survive the CHS procedure are included. The same reconstruction criteria are also used to create generator-level jets using generated stable particles (mean path length $c \tau>1 \mathrm{~cm}$, no minimum $p_{\mathrm{T}}$ requirement), removing neutrinos. Simulated pileup particles are not included in the generator-level jets. The ungroomed jet mass is taken as the mass of the four-momentum sum of the constituents of the AK8 jet without grooming applied. The groomed jet mass is taken as the mass of the four-momentum sum of the constituents of the AK8 jet with the SD algorithm applied. For this measurement, the $p_{\mathrm{T}}$ of the ungroomed AK8 jet is used for both the groomed and ungroomed jet cross sections, since it has been shown that the groomed jet $p_{\mathrm{T}}$ is not collinear-safe for the SD algorithm chosen [18].

Since only charged hadrons arising from pileup interactions are removed with the CHS procedure, corrections based on the jet area [41] are applied to the jet energies to remove the energy contribution of neutral hadrons from pileup. The area-based residual subtraction is not applied to the jet mass because it is observed [42] that this overcorrects the mass of hadronically decaying $\mathrm{W}$ bosons in a t $\overline{\mathrm{t}}$ control sample. Further jet energy scale (JES) corrections are applied as functions of $\eta$ and $p_{\mathrm{T}}$, derived from simulation. Data-tosimulation correction factors are also applied, using the methodology of ref. [42]. These corrections are also applied to the jet mass by scaling the mass by the same factor used for the $p_{\mathrm{T}}$ in the four-momentum of the jet.

To account for the poorer jet energy resolution (JER) in the data with respect to MC simulation, the reconstructed simulated jets are smeared compared to generator-level jets by the amounts listed in ref. [42]. The typical values of the JER are around 5-8\%. After these corrections, the JES and JER in MC simulation agree with those in data for both groomed and ungroomed jets.

The jet mass can also have differen $t$ scales and resolutions in experimental data and in MC simulation. In the MC simulation, the jet mass scale (JMS) and jet mass resolution (JMR) are respectively determined by comparing the means and widths of distributions of the jet mass at the reconstructed and generator levels $\left(m_{\text {reco }} / m_{\text {gen }}\right)$. The JMSs in MC simulation for groomed and ungroomed jets with our selection are shown in figure 1 as a 

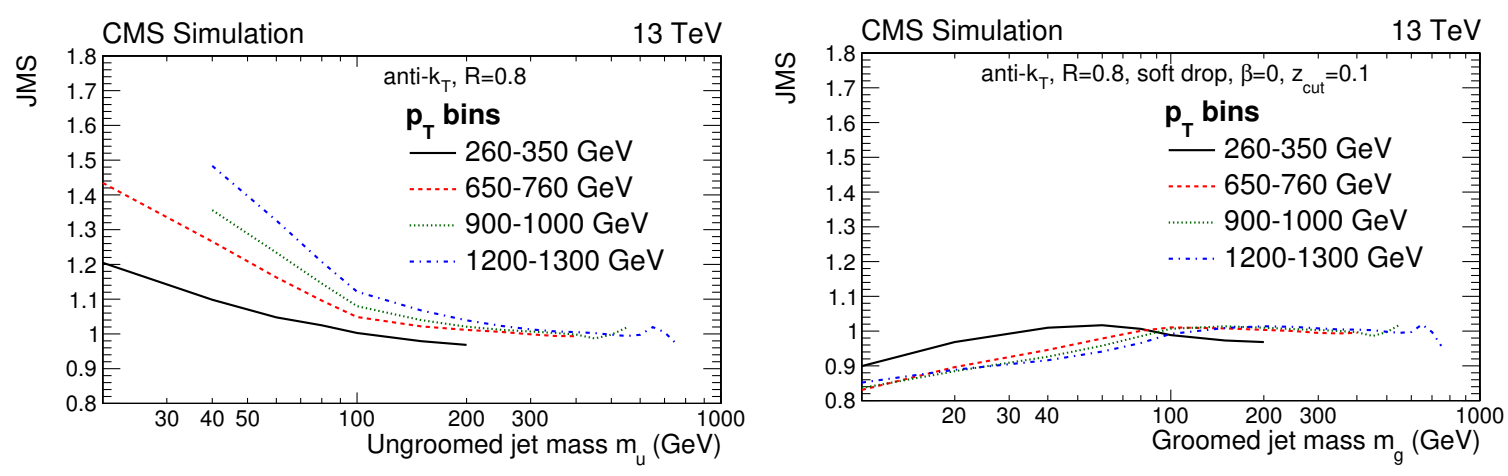

Figure 1. JMS in simulation (mean of a fit to $m_{\text {reco }} / m_{\text {gen }}$ ) for ungroomed (left) and groomed (right) jets in different generated $p_{\mathrm{T}}$ bins, as a function of the generated mass, with our selection. The correction to match the data is $0.98 \pm 0.01$. Uncertainties are not shown, for clarity, although statistical fluctuations at high masses near the splitting threshold for each $p_{\mathrm{T}}$ bin are not negligible.

function of the jet mass for various $p_{\mathrm{T}}$ bins, and similar distributions are shown for the JMR in figure 2. In these figures, finite-size effects of the jet can be observed at the highest masses in the lowest $p_{\mathrm{T}}$ bin. The JMS and JMR are accounted for directly in a detector response parameterization along with corrections to the $\mathrm{MC}$ simulation as to match data. The latter are derived using the approach outlined in ref. [43], except we use the SD algorithm to groom the jets, which is based on a fit of the jet mass from hadronically decaying $\mathrm{W}$ bosons in $\mathrm{t} \overline{\mathrm{t}}$ events where one $\mathrm{W}$ boson decays hadronically and the other decays leptonically (the "lepton plus jets" channel). The ratio of the fitted means in data and in MC simulation is the correction to the JMS, and the ratio of the fitted widths is the correction to the JMR. This estimate assumes that detector-related uncertainties will be the same for jets from $\mathrm{W}$ boson decays and jets initiated by quarks and gluons. The remaining differences are effects from the physics model, and are thus accounted for with our physics model uncertainty. The JMR and JMS corrections in the 2015 run of the LHC ("Run 2") are measured to be $1.07 \pm 0.12$ and $0.98 \pm 0.01$, respectively, using the methods of ref. [43]. In the 2010-2012 run of the LHC ("Run 1"), they were observed to be $1.11 \pm 0.09$ and $1.017 \pm 0.006$, respectively [44]. In addition to smearing the entire four-vector (including the $p_{\mathrm{T}}$ and the mass) of the jet by the JER, we also correct the jet mass distribution in the MC simulation to match the data in the $\mathrm{W}$ reference sample.

The data used in this analysis are from pp collisions collected in 2015 with 50 ns bunch spacing, as in ref. [45], as well as with $25 \mathrm{~ns}$ bunch spacing. The integrated luminosities of the 25 and $50 \mathrm{~ns}$ samples correspond to approximately 2.3 and $0.07 \mathrm{fb}^{-1}$, respectively. The data were collected with single-jet triggers of varying $p_{\mathrm{T}}$ thresholds as in the inclusive jet analysis from ref. [45]. As in that paper, the ensemble of events is constructed such that any given trigger only contributes to a specific $p_{\mathrm{T}}$ region, where it is fully efficient. However, since we are using a different $R$-parameter for the jet clustering algorithm (we use $R=0.8$ as opposed to $R=0.4$ and 0.7 ), our thresholds, shown in table 1 , are slightly different than those from the inclusive jet analysis [45]. The same $p_{\mathrm{T}}$ thresholds are used for both 50 and $25 \mathrm{~ns}$ data. In the collisions with $50 \mathrm{~ns}$ bunch spacing, the trigger prescales were 

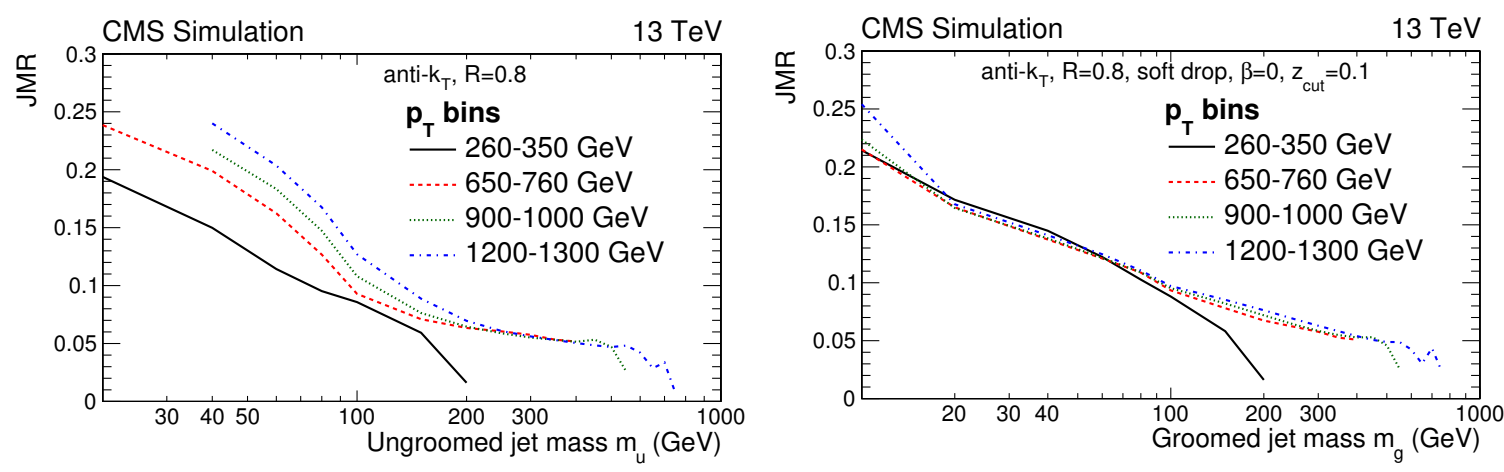

Figure 2. JMR in simulation (width of a fit to $m_{\text {reco }} / m_{\text {gen }}$ ) for ungroomed (left) and groomed (right) jets in different generated $p_{\mathrm{T}}$ bins, as a function of the generated mass, with our selection. The correction to match the data is $1.07 \pm 0.12$. Uncertainties are not shown, for clarity, although statistical fluctuations at high masses near the splitting threshold for each $p_{\mathrm{T}}$ bin are not negligible.

\begin{tabular}{|cc|}
\hline Trigger $p_{\mathrm{T}}$ threshold $(\mathrm{GeV})$ & $p_{\mathrm{T}}$ range $(\mathrm{GeV})$ \\
\hline 80 & $200-260$ \\
140 & $260-350$ \\
200 & $350-460$ \\
260 & $460-550$ \\
320 & $550-650$ \\
400 & $650-760$ \\
450 & $>760$ \\
\hline
\end{tabular}

Table 1. List of trigger thresholds used for each high-level trigger path. The ungroomed jet $p_{\mathrm{T}}$ ranges are shown in the second column. Events are selected such that exactly one fully efficient trigger is used for each $p_{\mathrm{T}}$ bin.

lower, and thus this dataset increases the number of events collected for $p_{\mathrm{T}}<460 \mathrm{GeV}$ by $10 \%$ compared to the $25 \mathrm{~ns}$ run alone, where the prescales are considerably higher. There are 43.9 million events in the $25 \mathrm{~ns}$ sample and 4.4 million events in the $50 \mathrm{~ns}$ sample.

\section{Analysis strategy}

The measurement of the dijet cross section is corrected for detector effects using an unfolding technique. The detector response is parameterized as a four-dimensional response matrix representing the migrations from reconstructed $m$ and $p_{\mathrm{T}}$ to generated $m$ and $p_{\mathrm{T}}$. This response matrix is used to correct the measured distribution, and the resulting unfolded distribution is compared to various theoretical models.

The events are required to have at least two jets, without an explicit third jet veto, which allows for an easier comparison to semi-analytical calculations. To ensure a highpurity dijet-like sample, we require that the asymmetry between the leading and subleading jet transverse momenta, defined as $p_{\mathrm{T} 1}$ and $p_{\mathrm{T}_{2}}$ respectively, satisfies $\left(p_{\mathrm{T}_{1}}-p_{\mathrm{T}_{2}}\right) /\left(p_{\mathrm{T} 1}+\right.$ 
$\left.p_{\mathrm{T} 2}\right)<0.3$ and the difference in azimuthal angles satisfies $\Delta\left(\phi_{1}-\phi_{2}\right)>\pi / 2$. In addition to ensuring a dijet topology, these criteria reduce the number of jets from detector noise. Both jets in the dijet system are included in the measurement.

All reconstructed AK8 jets are required to have $p_{\mathrm{T}}>200 \mathrm{GeV}$ and rapidity $|y|<2.4$, and jet identification requirements that remove detector noise outlined in ref. [46]. After the identification and topological requirements, the number of jets from detector noise in this sample is negligible. Generator-level AK8 jets are required to have $|y|<2.4$ and a very low $p_{\mathrm{T}}$ selection $(>50 \mathrm{GeV})$ to ensure an accurate estimate of the migration of events between $p_{\mathrm{T}}$ bins, but the measurement is presented only for $p_{\mathrm{T}}>200 \mathrm{GeV}$ at the generator level. The $p_{\mathrm{T}}$ asymmetry and $\Delta \phi$ selections are also applied at the generator level, but the $p_{\mathrm{T}}$ asymmetry is a slowly varying function of $p_{\mathrm{T}}$, so this does not strongly affect the $p_{\mathrm{T}}$ migration.

The bins in ungroomed jet $p_{\mathrm{T}}$ are chosen so that each bin is populated by exactly one trigger, with binning as shown in table 1 (i.e., the trigger with a threshold of $80 \mathrm{GeV}$ will be used to populate the $200-260 \mathrm{GeV}$ bin, etc). Above the threshold of the lowest unprescaled trigger at $p_{\mathrm{T}}=760 \mathrm{GeV}$, more uniform $p_{\mathrm{T}}$ binning is used. The lower bin boundaries are $200,260,350,460,550,650,760,900,1000,1100,1200,>1300 \mathrm{GeV}$. The choice of jet mass bins is a trade-off among the approximate detector resolution of $\sim 10 \mathrm{GeV}$ [42], the purity (the fraction of events in a reconstructed bin that are generated in the same bin), and the stability (the fraction of events in a generated bin that are reconstructed in the same bin). The lower bin boundaries are 10,20,40,60,80,100 GeV and then increase by $50 \mathrm{GeV}$ beyond $100 \mathrm{GeV}$. The same binning is used for both the reconstructed and generated jet mass. There are, however, kinematic regions that have insufficient accuracy for the jet mass measurement. This inaccuracy can arise from the finite spatial resolution of the detector or from excessive pileup contamination. These regions in $p_{\mathrm{T}}$ and $m$ are removed by requiring that the uncertainty in a Gaussian fit to the distribution of $m_{\text {reco }} / m_{\text {gen }}$ in MC simulation is less than $50 \%$ for that region. We do not consider the measurements below those thresholds. For ungroomed jets, the dominant effect is pileup contamination, and we do not consider jet masses below $20 \mathrm{GeV}$ when $p_{\mathrm{T}}<760 \mathrm{GeV}$, or below $40 \mathrm{GeV}$ when $p_{\mathrm{T}}>760 \mathrm{GeV}$. For groomed jets, the dominant effect is the finite spatial resolution of the detector, and we do not consider jet masses below $10 \mathrm{GeV}$ for all $p_{\mathrm{T}}$ bins.

The unfolding procedure of D'Agostini with early stopping is applied [47-49]. The detector response is from $\mathrm{MC}$ simulation, and parameterized in bins of ungroomed jet $p_{\mathrm{T}}$ and ungroomed jet mass for the ungroomed case, and in bins of ungroomed jet $p_{\mathrm{T}}$ and groomed jet mass for the groomed case. The central value of the response matrix is constructed using PYTHIA8. The uncertainty in the response matrix is estimated by varying the systematic uncertainties described below, rederiving the matrix for each case, and applying the modified response matrix to the reconstructed data, taking the difference in the unfolded distribution as the resultant uncertainty.

The number of iterations in the early stopping procedure has been optimized as follows. The response matrix is used to unfold the PYTHIA 8 MC simulation varying the number of iterations in the regularization. A simple matrix multiplication is then used to refold the unfolded MC simulation. The resulting refolded distribution is compared to the original 
reconstructed distribution and a $\chi^{2}$ probability is calculated. The optimal number of iterations is determined as the lowest number such that the $\chi^{2}$ probability exceeded $99 \%$. We found $n=3$ to be optimal. Varying the number of iterations did not lead to a significant change in the results.

The uncertainties in the unfolded result due to statistical uncertainties in the MC simulation are nontrivial to estimate in the unfolding procedure because the events have different weights, so the statistical uncertainty is not correctly estimated by simply adding the uncertainties from weighted events in quadrature. Instead of standard methods, we employ a "jackknife resampling" technique (a linear bootstrapping method) [50, 51], where we create 10 samples, each with $90 \%$ of the MC events (such that each event is excluded from exactly one resampling), and unfold the spectrum. The root-mean-square of this ensemble of distributions, multiplied by $\sqrt{10 / 9}$ to account for using $90 \%$ of the events per iteration, is taken as the statistical uncertainty in the MC simulation.

There are both theoretical and experimental systematic uncertainties that affect this measurement, along with uncertainties from the unfolding procedure. The uncertainties are estimated by creating response matrices with the systematic effect applied, and unfolding the reconstructed data to account for systematic migrations. Unless otherwise specified, the difference between the systematically varied output and the nominal output is taken to be the systematic uncertainty for that source. The uncertainties from different sources are then added together in quadrature.

The experimental uncertainties that affect the measurement are the JES, JER, JMS, JMR, pileup, and luminosity. The theoretical uncertainties include uncertainties in the physics model and PDFs.

The JES uncertainty is evaluated by varying the jet energy and mass up and down by the appropriate uncertainties for all of the JES uncertainty sources as described in ref. [42]. The JER uncertainty is evaluated by smearing the jet energy and mass by an additional factor as described in ref. [42]. The central value is determined by correcting the MC simulation to match the JER from data, and the uncertainties are evaluated by correcting the MC simulation by the JER systematic variations. This is also propagated to the jet mass because these effects are correlated for jets reconstructed from PF candidates. The uncertainty due to the finite resolution of the jet angle is found to be negligible compared to other uncertainties.

As mentioned in section 3 , the JMR in simulation is corrected by $1.07 \pm 0.12$ to match the measured difference between data and MC simulation for the mass of $\mathrm{W}$ boson jets in lepton + jets $t \bar{t}$ events [52]. The JMS in MC simulation approaches unity for larger jet $p_{\mathrm{T}}$ and masses. Deviations from unity are included directly in the response matrix, as described in section 3 .

The pileup uncertainty is estimated by reweighting the pileup distribution of the MC simulation up and down, assuming a change of the minimum bias cross section by $2.7 \%$ [53]. For the absolute cross section, the luminosity uncertainty of $2.7 \%$ is also taken as an additional systematic uncertainty.

The PDF uncertainty is taken from the PDF4LHC15_NLO_100 meta-PDF set using the PYTHIA8 generator. The physics model uncertainty is evaluated by comparing a response 

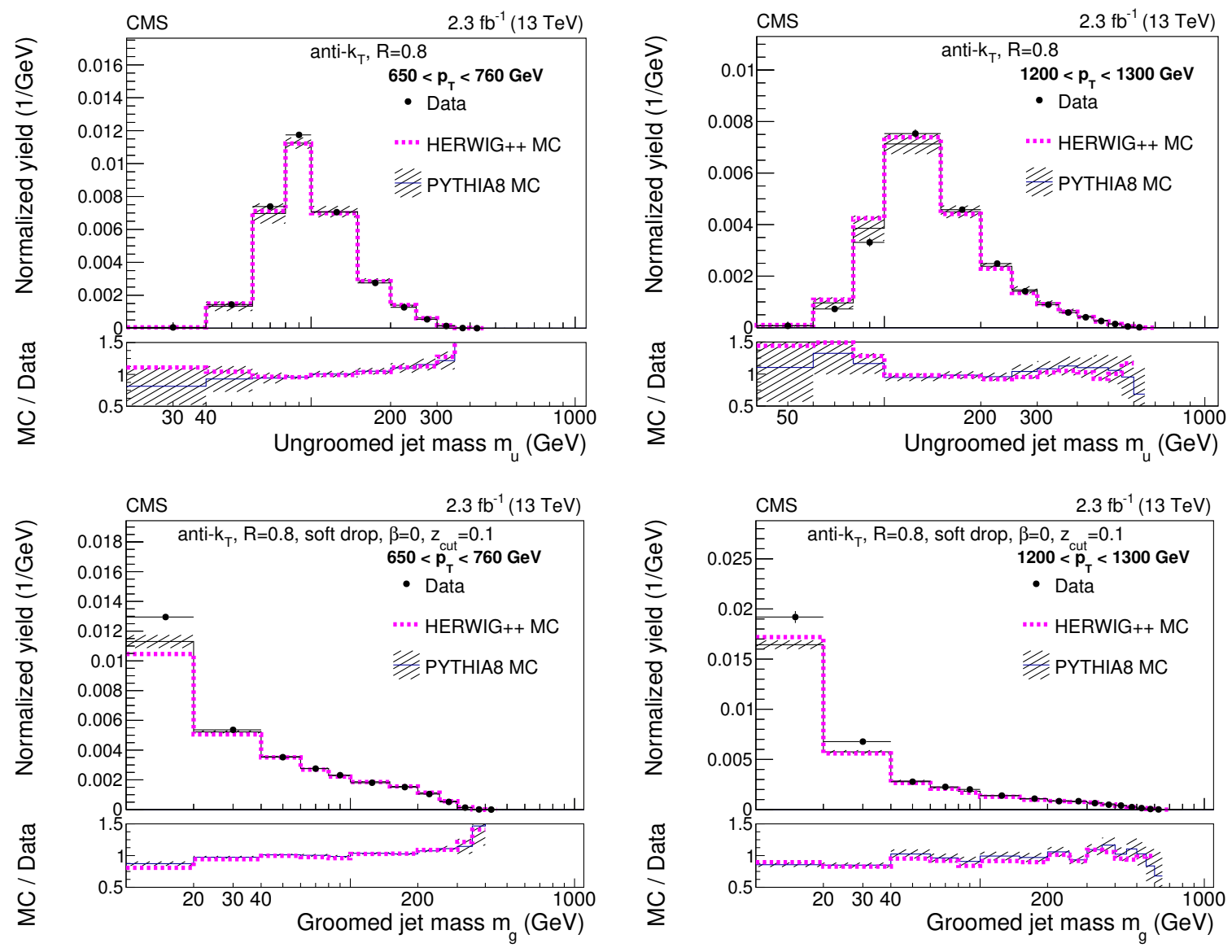

Figure 3. Comparison of data to MC simulation for ungroomed jets (top) and groomed jets (bottom) for two $p_{\mathrm{T}}$ bins at the detector level. The data and their statistical uncertainties are shown by the black points with the error bars. The PYTHIA 8 results before the unfolding are shown in the shaded histograms, including experimental and theoretical systematic uncertainties. The HERWIG ++ results before unfolding are shown without uncertainties as a dashed magenta histogram.

matrix derived from PYTHIA 8 to a response matrix derived from HERWIG ++ . The difference between the two results for the two cases is taken as the systematic uncertainty.

The jet mass distributions in data before unfolding are compared to PYTHIA 8 and HER$\mathrm{WIG}++\mathrm{MC}$ simulations in figure 3. The uncertainties before the unfolding are evaluated by varying the MC simulation up and down by each systematic effect described in the previous paragraphs, and using the sum of the differences to the nominal case in quadrature as the uncertainty band. The MC simulation distributions include the theoretical systematic uncertainties, the experimental systematic uncertainties, and the statistical uncertainties of the MC samples. The data distributions include only the statistical uncertainties. These data distributions are unfolded to obtain the cross section measurements.

Figure 4 shows the uncertainties in the absolute cross section for all of the various sources listed above, while figure 5 shows the same uncertainties in the normalized cross section. The jet energy uncertainties in the normalized cross sections are smaller than 

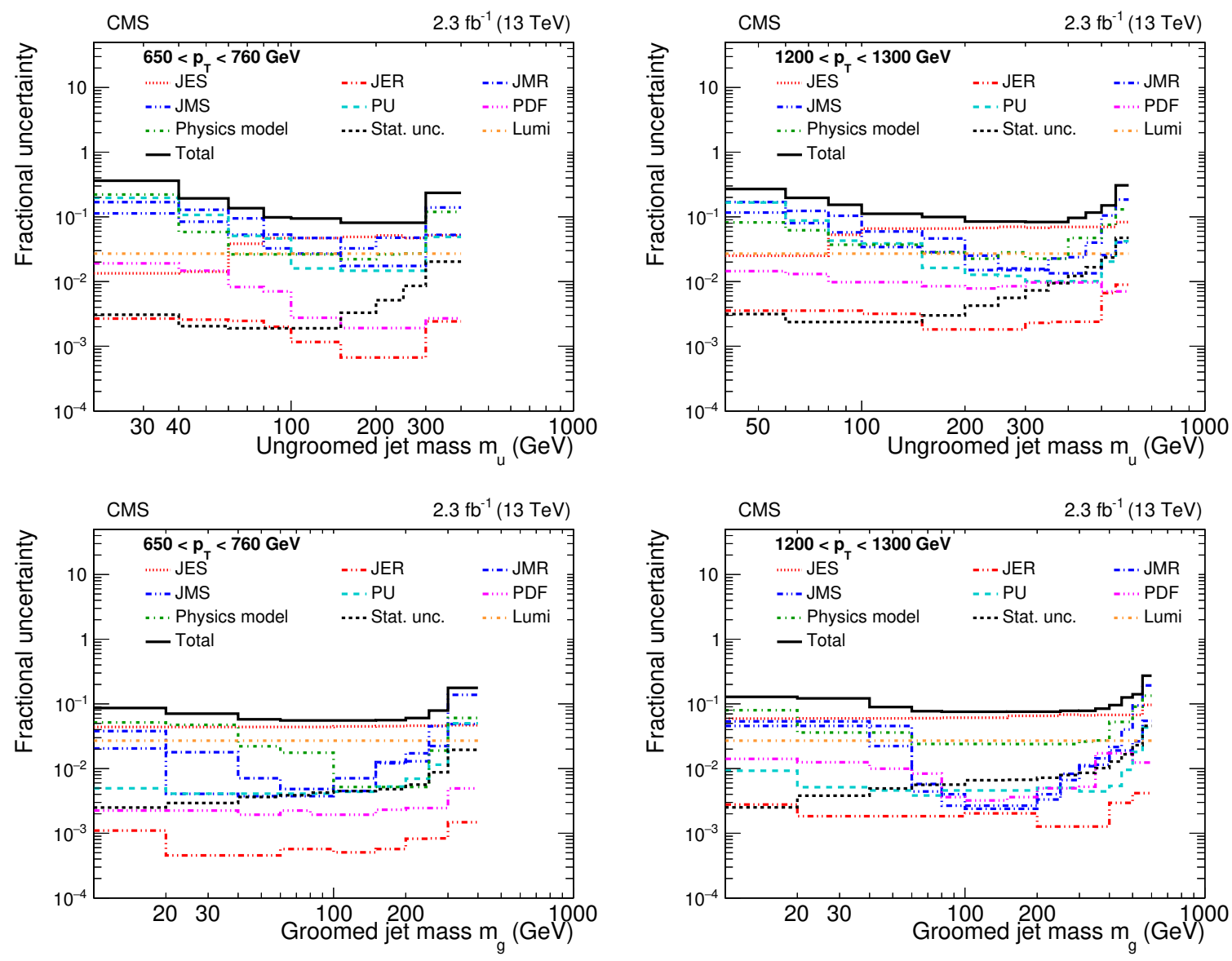

Figure 4. Systematic uncertainties in the absolute cross section after unfolding for two $p_{\mathrm{T}}$ bins before grooming (top) and after grooming (bottom). The red dotted lines show the JES uncertainties. The red dash-dot-dotted lines show the JER uncertainties. The blue dash-dotted lines show the JMR uncertainties. The blue dash-dot-dotted lines show the JMS uncertainties. The cyan dashed lines show the PU uncertainties. The orange dash-dotted lines show the luminosity uncertainties. The magenta dash-dot-dot-dotted lines show the PDF uncertainties. The green dashdotted lines show the physics model uncertainties. The black short-dashed lines show the statistical uncertainties.

in the absolute cross sections because the distributions are normalized per $p_{\mathrm{T}}$ bin. The uncertainties at low jet mass are dominated by the physics model, JMS, JMR, and pileup uncertainties for the ungroomed jets. However, the grooming procedure dramatically reduces the physics model uncertainties by removing the soft components that are difficult to model, as well as the pileup uncertainty, since pileup mimics soft radiation. At higher jet mass, the jets are at the threshold of splitting into two, so the uncertainties increase because of $p_{\mathrm{T}}$ bin migration. In addition, the phase space is severely restricted in this regime, so the statistical uncertainty also grows. 

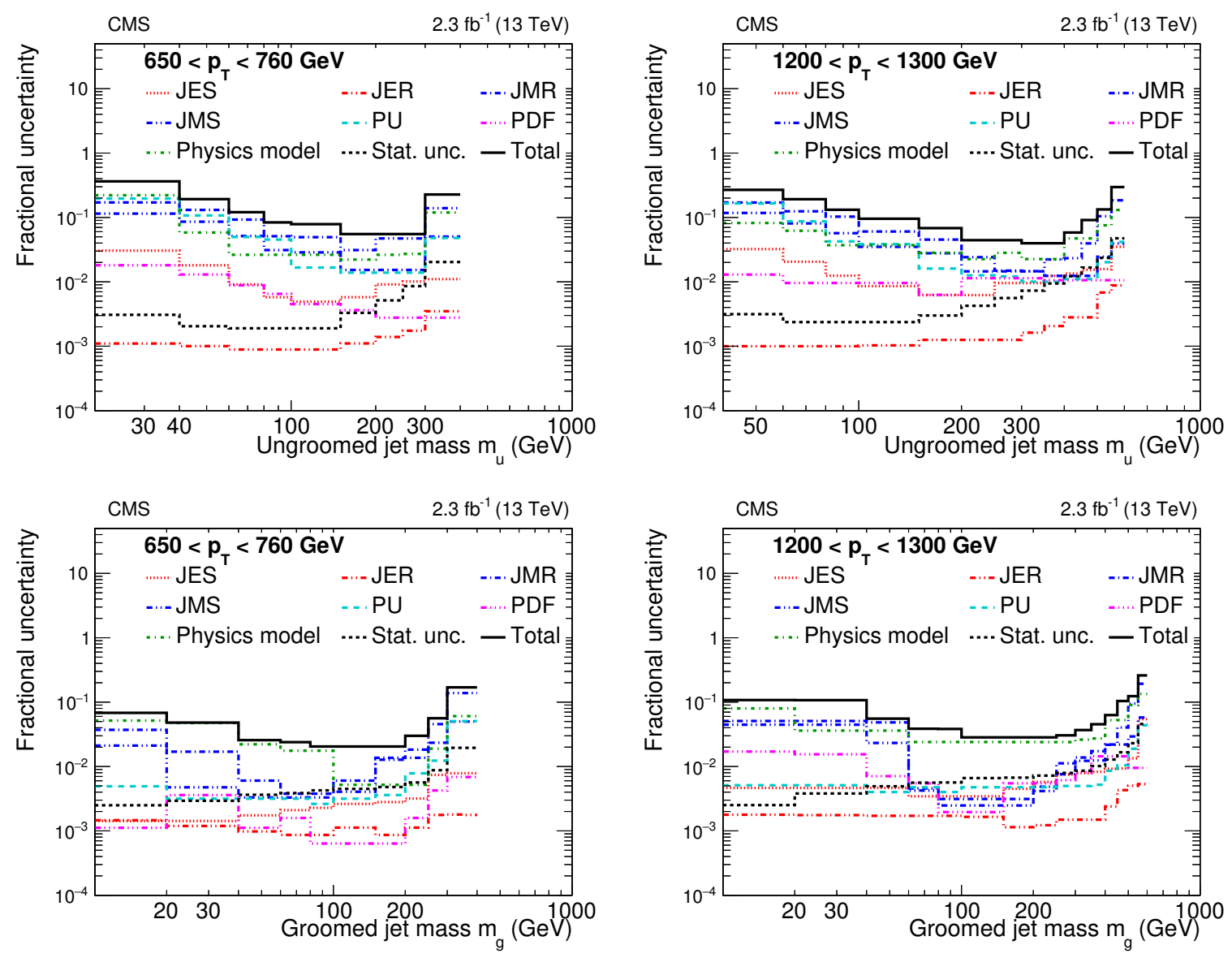

Figure 5. Systematic uncertainties in the normalized cross section after unfolding for two $p_{\mathrm{T}}$ bins before grooming (top) and after grooming (bottom). The red dotted lines show the JES uncertainties. The red dash-dot-dotted lines show the JER uncertainties. The blue dash-dotted lines show the JMR uncertainties. The blue dash-dot-dotted lines show the JMS uncertainties. The cyan dashed lines show the PU uncertainties. The orange dash-dotted lines show the luminosity uncertainties. The magenta dash-dot-dot-dotted lines show the PDF uncertainties. The green dashdotted lines show the physics model uncertainties. The black short-dashed lines show the statistical uncertainties.

\section{Results}

The absolute cross sections as a function of the jet mass are shown for two $p_{\mathrm{T}}$ bins in figure 6. The cross sections are compared to PYTHIA8, POWHEG + PYTHIA8, and HERWIG ++ predictions, which are scaled to match the number of events in the data.

The normalized cross sections as a function of the jet mass are shown for two $p_{\mathrm{T}}$ bins in figure 7. All $p_{\mathrm{T}}$ bins of the normalized cross section are shown in figures 8 and 9 . The lowest masses in the measurement are difficult to measure experimentally, as described in section 4. As mentioned there, the mass measurements are presented for ungroomed jets for $m>20 \mathrm{GeV}$ when $p_{\mathrm{T}}<760 \mathrm{GeV}$, and $m>40 \mathrm{GeV}$ for jets with $p_{\mathrm{T}}>760 \mathrm{GeV}$. Results for groomed jets are presented for $m>10 \mathrm{GeV}$ for all $p_{\mathrm{T}}$. The normalized results for the 

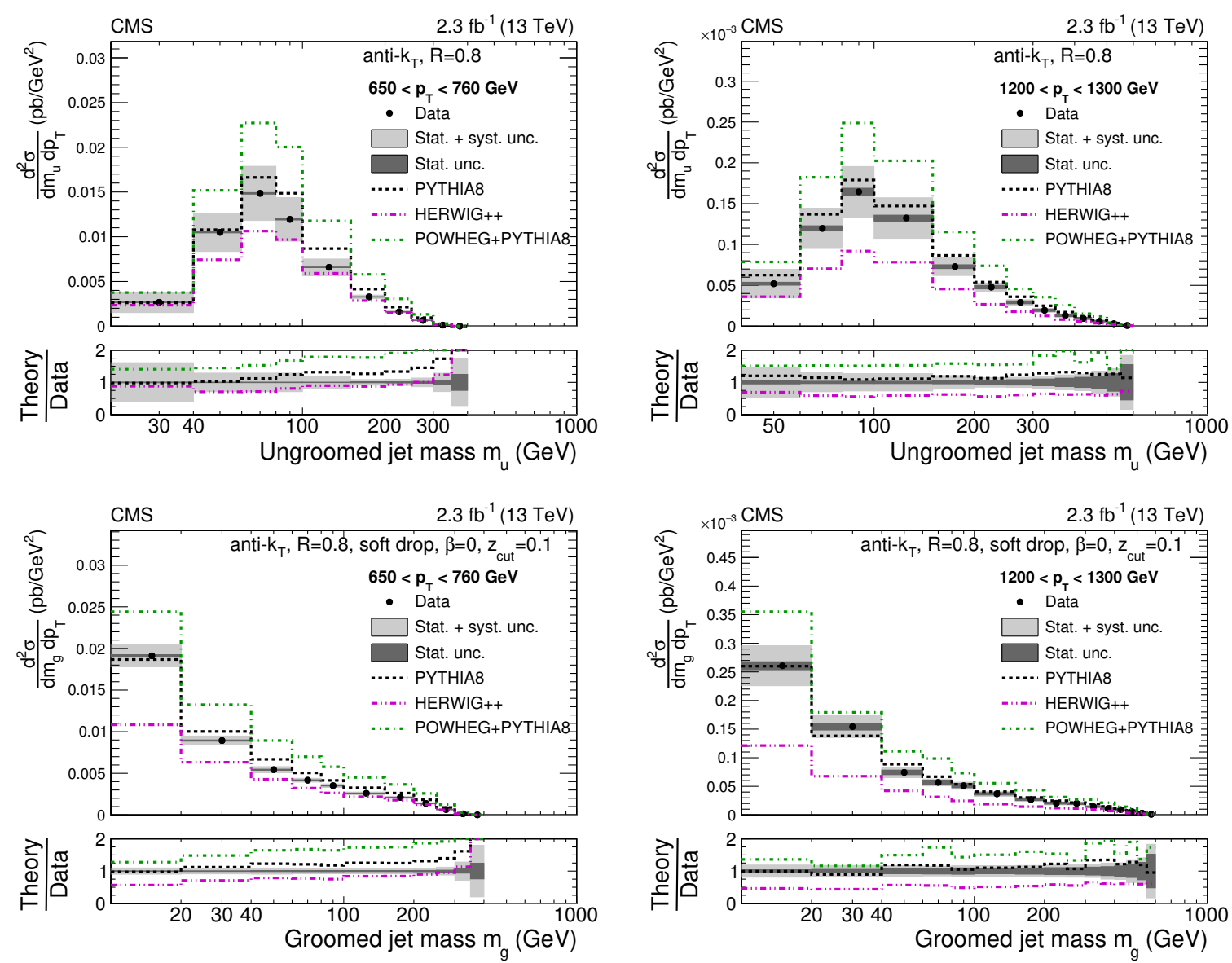

Figure 6. Absolute cross section for ungroomed (top) and groomed (bottom) jets for two $p_{\mathrm{T}}$ bins. The data are shown by the black points, with dark grey bands for the statistical uncertainty (Stat. unc.) and with light grey bands for the total uncertainty (Stat. + sys. unc., added in quadrature). The predictions from PYTHIA8, HERWIG ++, and POWHEG + PYTHIA are shown with dashed black, dash-dot-dotted magenta, and dash-dotted green histograms, respectively, with no uncertainties shown. The MC simulations are normalized to the data over the entire $p_{\mathrm{T}}$ range.

groomed jets are also compared to the calculation of the jet mass from refs. [17] and [18]. The normalized results are scaled to unity for each individual jet $p_{\mathrm{T}}$ bin for the unfolded data, for the PYTHIA8, HERWIG ++, POWHEG + PYTHIA8 predictions, and for the results from the calculation from the authors of ref. [18]. The calculation from ref. [17], on the other hand, is normalized to match the data at $50 \mathrm{GeV}$, since the calculation does not consider nonperturbative corrections that are important below that value. As a reminder, the prediction from ref. [17] includes nonperturbative effects as an uncertainty, while the prediction from ref. [18] directly includes nonperturbative effects in the prediction, and has smaller systematic uncertainties in low-mass regions where nonperturbative effects are important.

Comparing the normalized ungroomed and groomed cross sections (figure 7), our results show that the grooming algorithm considerably lowers the jet mass and suppresses the Sudakov peak, as expected. The precision of the measurement improves, since the grooming algorithm removes the parts of the jet arising from soft radiation and the pileup 

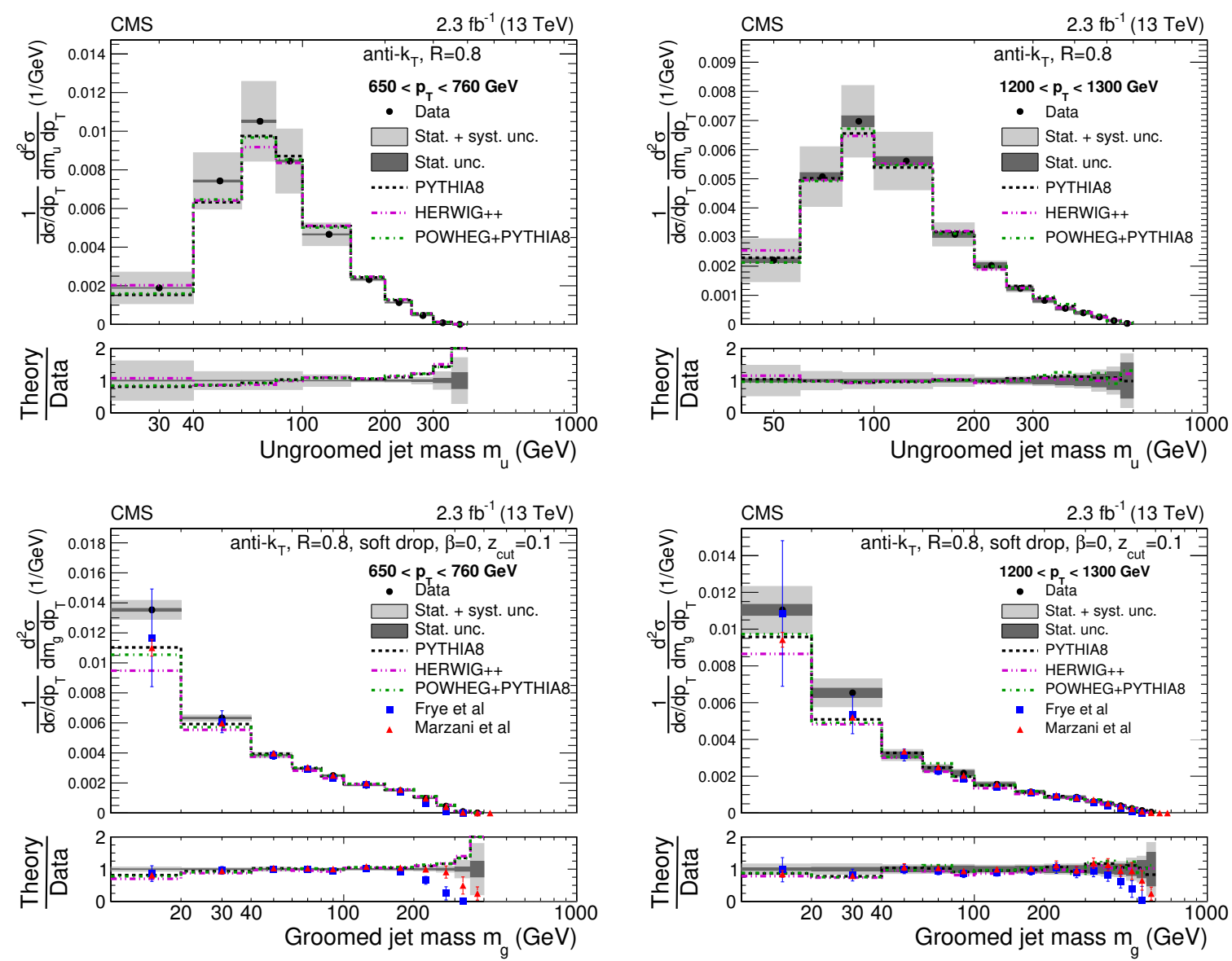

Figure 7. Normalized cross section for the ungroomed (top) and groomed (bottom) jets for two $p_{\mathrm{T}}$ bins. The predictions from PYTHIA8, HERWIG ++ , and POWHEG + PYTHIA are shown with dashed black, dash-dot-dotted magenta, and dash-dotted green histograms, respectively, with no uncertainties shown. The predictions from ref. [17] (Frye et al.) are shown with blue squares. The uncertainties include scale variations and an estimate of nonperturbative effects. The predictions from ref. [18] (Marzani et al.) are shown with red triangles. The uncertainties include only effects from scale variations, since nonperturbative corrections have been considered directly in the calculation. Both predictions diverge from the data at high mass due to fixed-order matching.

contribution. Figure 5 shows the reduced effect of the uncertainties from the physics model and pileup, decreasing the overall uncertainty from $\sim 8-12 \%$ at $m=100 \mathrm{GeV}$ for ungroomed jets to $\sim 2-5 \%$ for groomed jets at the same mass. The absolute cross sections (figure 6) have larger uncertainties than the normalized cross sections because of jet energy uncertainties (JES and JER), as shown in figure 4.

The LO theory predictions with a MC-based physics model are from PYTHIA8 and HERWIG ++ , and they predict the ungroomed jet mass measurement within uncertainties, although the experimental uncertainties are large. Above the splitting threshold $\left(m / p_{\mathrm{T}}>\right.$ 0.3 ), the prediction is slightly larger than the data. After grooming, the experimental precision improves, so some deviations from the data can be observed at very low jet masses. Some disagreements are observed between PYTHIA 8 and HERWIG ++ . The PYTHIA 8 


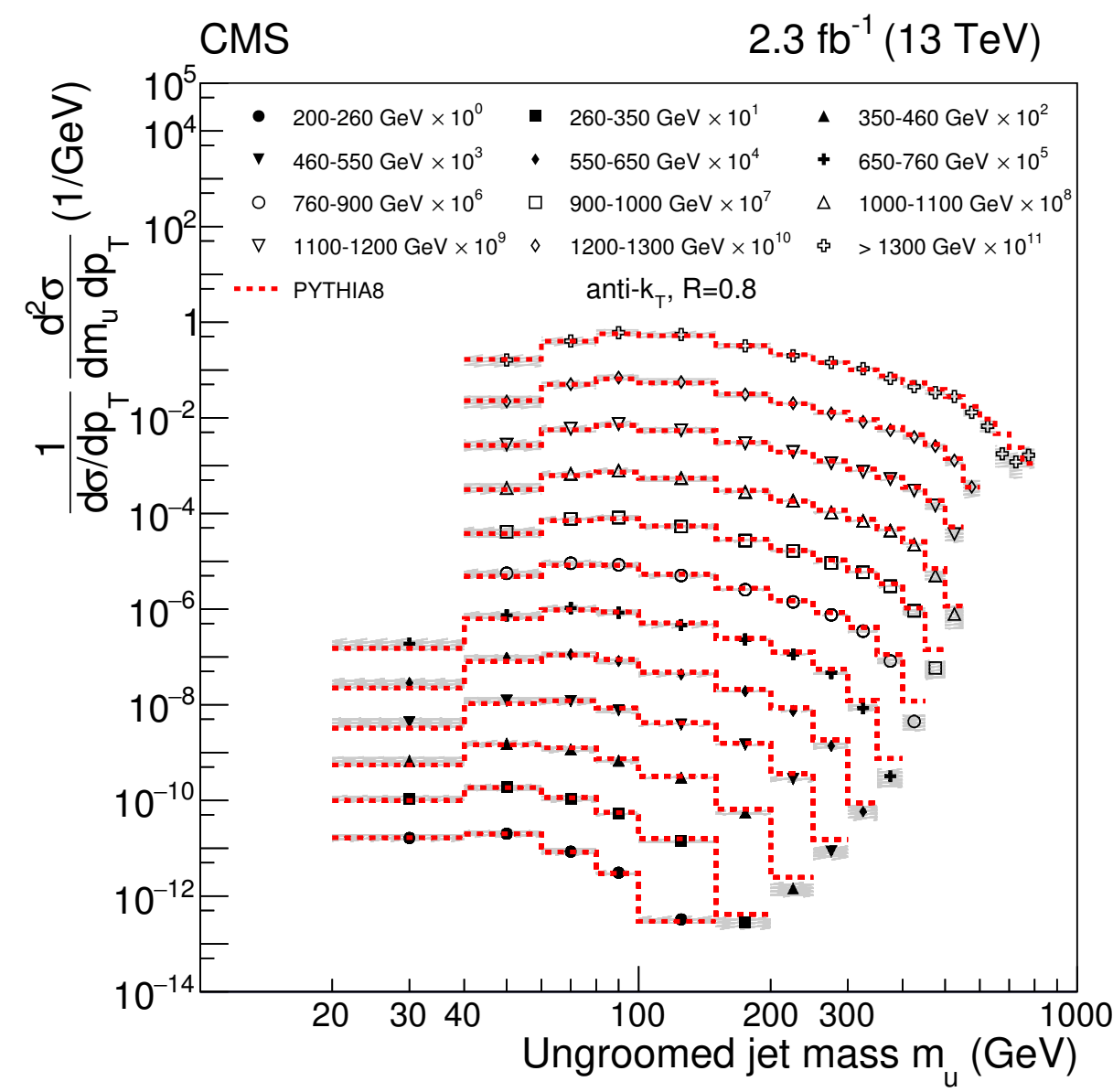

Figure 8. Normalized cross section for ungroomed jets for all $p_{\mathrm{T}}$ bins. Bins with statistical uncertainty larger than $60 \%$ are not shown. The data are shown with markers for each $p_{\mathrm{T}}$ bin, scaled by a factor for better visibility. The total uncertainties (statistical added to systematic in quadrature) are shown with grey bands. The predictions from PYTHIA 8 are shown as a dashed red line.

generator tends to predict the data better, although for $m / p_{\mathrm{T}}<0.1$, the agreement is worse than in the range $0.1<m / p_{\mathrm{T}}<0.3$. The same disagreement above the splitting threshold is observed as in the ungroomed case. The NLO theory prediction with a MC-based physics model is from POWHEG + PYTHIA8, and is observed to have largely the same behavior as PYTHIA8 alone, so the largest effects are coming from the physics model.

The LO and NLO theory predictions with analytic resummation (refs. [17] and [18], respectively) agree overall with the data, with some slight disagreements at very low masses. For $m / p_{\mathrm{T}}>0.3$, the fixed-order matrix element merging is insufficient to capture the true dynamics due to kinematic effects, as described in section 2 , so the predictions diverge from the data. They agree with the predictions using MC-based physics models, although some disagreement is observed at very low masses. The resummed predictions are slightly closer to the PYTHIA8 prediction than to the HERWIG ++ one. 


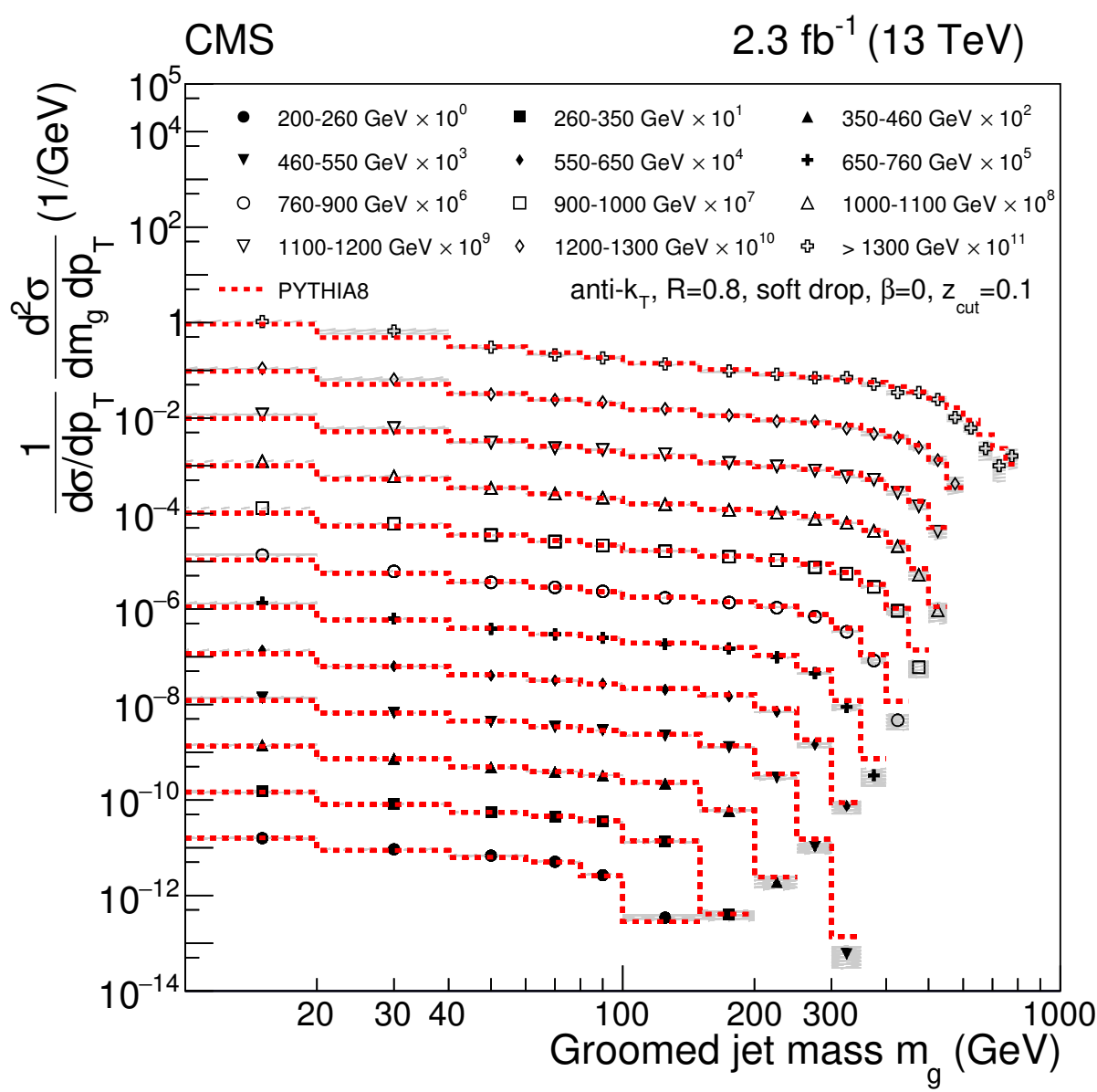

Figure 9. Normalized cross section for groomed jets for all $p_{\mathrm{T}}$ bins. Bins with statistical uncertainty larger than $60 \%$ are not shown. The data are shown with markers for each $p_{\mathrm{T}}$ bin, scaled by a factor for better visibility. The total uncertainties (statistical added to systematic in quadrature) are shown with grey bands. The predictions from PYTHIA 8 are shown as a dashed red line.

\section{Summary}

The double-differential production cross section has been presented for dijet events in bins of ungroomed jet $p_{\mathrm{T}}$ as a function of the jet mass, with and without a jet grooming algorithm applied. The grooming algorithm is observed to decrease the jet mass overall, and reduce the sensitivity of the observable to details of the physics modeling and pileup effects. The data are compared to various theoretical predictions, including new calculations using analytical resummation. Overall, in the normalized cross section, the theoretical predictions agree with the measured cross sections within the uncertainties for masses from 10 to $30 \%$ of the jet transverse momentum. 


\section{Acknowledgments}

We congratulate our colleagues in the CERN accelerator departments for the excellent performance of the LHC and thank the technical and administrative staffs at CERN and at other CMS institutes for their contributions to the success of the CMS effort. In addition, we gratefully acknowledge the computing centres and personnel of the Worldwide LHC Computing Grid for delivering so effectively the computing infrastructure essential to our analyses. Finally, we acknowledge the enduring support for the construction and operation of the LHC and the CMS detector provided by the following funding agencies: BMWFW and FWF (Austria); FNRS and FWO (Belgium); CNPq, CAPES, FAPERJ, FAPERGS, and FAPESP (Brazil); MES (Bulgaria); CERN; CAS, MoST, and NSFC (China); COLCIENCIAS (Colombia); MSES and CSF (Croatia); RPF (Cyprus); SENESCYT (Ecuador); MoER, ERC IUT, and ERDF (Estonia); Academy of Finland, MEC, and HIP (Finland); CEA and CNRS/IN2P3 (France); BMBF, DFG, and HGF (Germany); GSRT (Greece); NKFIA (Hungary); DAE and DST (India); IPM (Iran); SFI (Ireland); INFN (Italy); MSIP and NRF (Republic of Korea); MES (Latvia); LAS (Lithuania); MOE and UM (Malaysia); BUAP, CINVESTAV, CONACYT, LNS, SEP, and UASLP-FAI (Mexico); MOS (Montenegro); MBIE (New Zealand); PAEC (Pakistan); MSHE and NSC (Poland); FCT (Portugal); JINR (Dubna); MON, RosAtom, RAS, RFBR, and NRC KI (Russia); MESTD (Serbia); SEIDI, CPAN, PCTI, and FEDER (Spain); MOSTR (Sri Lanka); Swiss Funding Agencies (Switzerland); MST (Taipei); ThEPCenter, IPST, STAR, and NSTDA (Thailand); TUBITAK and TAEK (Turkey); NASU and SFFR (Ukraine); STFC (United Kingdom); DOE and NSF (U.S.A.).

Individuals have received support from the Marie-Curie programme and the European Research Council and Horizon 2020 Grant, contract No. 675440 (European Union); the Leventis Foundation; the A. P. Sloan Foundation; the Alexander von Humboldt Foundation; the Belgian Federal Science Policy Office; the Fonds pour la Formation à la Recherche dans l'Industrie et dans l'Agriculture (FRIA-Belgium); the Agentschap voor Innovatie door Wetenschap en Technologie (IWT-Belgium); the F.R.S.-FNRS and FWO (Belgium) under the "Excellence of Science - EOS" - be.h project n. 30820817; the Ministry of Education, Youth and Sports (MEYS) of the Czech Republic; the Lendület ("Momentum") Programme and the János Bolyai Research Scholarship of the Hungarian Academy of Sciences, the New National Excellence Program ÚNKP, the NKFIA research grants 123842, 123959, 124845, 124850 and 125105 (Hungary); the Council of Science and Industrial Research, India; the HOMING PLUS programme of the Foundation for Polish Science, cofinanced from European Union, Regional Development Fund, the Mobility Plus programme of the Ministry of Science and Higher Education, the National Science Center (Poland), contracts Harmonia 2014/14/M/ST2/00428, Opus 2014/13/B/ST2/02543, 2014/15/B/ST2/03998, and 2015/19/B/ST2/02861, Sonata-bis 2012/07/E/ST2/01406; the National Priorities Research Program by Qatar National Research Fund; the Programa Estatal de Fomento de la Investigación Científica y Técnica de Excelencia María de Maeztu, grant MDM-2015-0509 and the Programa Severo Ochoa del Principado de Asturias; the Thalis and Aristeia programmes cofinanced by EU-ESF and the Greek NSRF; the Rachadapisek Sompot Fund for 
Postdoctoral Fellowship, Chulalongkorn University and the Chulalongkorn Academic into Its 2nd Century Project Advancement Project (Thailand); the Welch Foundation, contract C-1845; and the Weston Havens Foundation (U.S.A.).

We would like to thank Christopher Frye, Andrew Larkoski, Simone Marzani, Gavin Salam, Matthew Schwartz, Lais Schunk, Gregory Soyez, and Kai Yan for providing calculations of theoretical predictions of the jet mass distribution for this analysis.

Open Access. This article is distributed under the terms of the Creative Commons Attribution License (CC-BY 4.0), which permits any use, distribution and reproduction in any medium, provided the original author(s) and source are credited.

\section{References}

[1] S. Höche, Introduction to parton-shower event generators, in Proceedings, Theoretical Advanced Study Institute in Elementary Particle Physics: Journeys Through the Precision Frontier: Amplitudes for Colliders (TASI 2014), Boulder, Colorado, June 2-27, 2014, pp. 235-295 (2015) [DOI:10.1142/9789814678766_0005] [arXiv:1411.4085] [INSPIRE].

[2] A. Buckley et al., General-purpose event generators for LHC physics, Phys. Rept. 504 (2011) 145 [arXiv: 1101.2599$]$ [INSPIRE].

[3] A. Abdesselam et al., Boosted objects: A probe of beyond the standard model physics, Eur. Phys. J. C 71 (2011) 1661 [arXiv:1012.5412] [InSPIRE].

[4] A. Altheimer et al., Boosted objects and jet substructure at the LHC. Report of BOOST2012, held at IFIC Valencia, 23rd-27th of July 2012, Eur. Phys. J. C 74 (2014) 2792 [arXiv: 1311.2708] [INSPIRE].

[5] D. Adams et al., Towards an understanding of the correlations in jet substructure, Eur. Phys. J. C 75 (2015) 409 [arXiv:1504.00679] [INSPIRE].

[6] M. Dasgupta, A. Fregoso, S. Marzani and G.P. Salam, Towards an understanding of jet substructure, JHEP 09 (2013) 029 [arXiv: 1307.0007] [INSPIRE].

[7] CMS collaboration, Studies of jet mass in dijet and $W / Z+$ jet events, JHEP 05 (2013) 090 [arXiv: 1303.4811] [INSPIRE].

[8] M. Dasgupta and G.P. Salam, Resummation of nonglobal QCD observables, Phys. Lett. B 512 (2001) 323 [hep-ph/0104277] [INSPIRE].

[9] S. Catani, L. Trentadue, G. Turnock and B.R. Webber, Resummation of large logarithms in $e^{+} e^{-}$event shape distributions, Nucl. Phys. B 407 (1993) 3 [INSPIRE].

[10] A. Banfi, G.P. Salam and G. Zanderighi, Semi-numerical resummation of event shapes, JHEP 01 (2002) 018 [hep-ph/0112156] [INSPIRE].

[11] A. Banfi, G.P. Salam and G. Zanderighi, Phenomenology of event shapes at hadron colliders, JHEP 06 (2010) 038 [arXiv: 1001.4082] [INSPIRE].

[12] A.J. Larkoski, S. Marzani, G. Soyez and J. Thaler, Soft drop, JHEP 05 (2014) 146 [arXiv: 1402.2657] [INSPIRE].

[13] M. Dasgupta, A. Fregoso, S. Marzani and A. Powling, Jet substructure with analytical methods, Eur. Phys. J. C 73 (2013) 2623 [arXiv:1307.0013] [INSPIRE].

[14] M. Dasgupta, A. Powling and A. Siodmok, On jet substructure methods for signal jets, JHEP 08 (2015) 079 [arXiv: 1503.01088] [INSPIRE]. 
[15] A.J. Larkoski, I. Moult and D. Neill, Non-global logarithms, factorization and the soft substructure of jets, JHEP 09 (2015) 143 [arXiv:1501.04596] [INSPIRE].

[16] J.M. Butterworth, A.R. Davison, M. Rubin and G.P. Salam, Jet substructure as a new Higgs search channel at the LHC, Phys. Rev. Lett. 100 (2008) 242001 [arXiv:0802.2470] [INSPIRE].

[17] C. Frye, A.J. Larkoski, M.D. Schwartz and K. Yan, Factorization for groomed jet substructure beyond the next-to-leading logarithm, JHEP 07 (2016) 064 [arXiv:1603.09338] [INSPIRE].

[18] S. Marzani, L. Schunk and G. Soyez, A study of jet mass distributions with grooming, JHEP 07 (2017) 132 [arXiv:1704.02210] [INSPIRE].

[19] ATLAS collaboration, Measurement of the soft-drop jet mass in pp collisions at $\sqrt{s}=13 \mathrm{TeV}$ with the ATLAS detector, Phys. Rev. Lett. 121 (2018) 092001 [arXiv: 1711.08341] [INSPIRE].

[20] T. Sjöstrand et al., An introduction to PYTHIA 8.2, Comput. Phys. Commun. 191 (2015) 159 [arXiv: 1410.3012] [INSPIRE].

[21] CMS collaboration, Event generator tunes obtained from underlying event and multiparton scattering measurements, Eur. Phys. J. C 76 (2016) 155 [arXiv:1512.00815] [INSPIRE].

[22] NNPDF collaboration, R.D. Ball et al., Parton distributions for the LHC Run II, JHEP 04 (2015) 040 [arXiv: 1410.8849] [INSPIRE].

[23] A. Buckley et al., LHAPDF6: parton density access in the LHC precision era, Eur. Phys. J. C 75 (2015) 132 [arXiv:1412.7420] [INSPIRE].

[24] J. Butterworth et al., PDF4LHC recommendations for LHC Run II, J. Phys. G 43 (2016) 023001 [arXiv: 1510.03865] [INSPIRE].

[25] M. Bahr et al., HERWIG++ physics and manual, Eur. Phys. J. C 58 (2008) 639 [arXiv: 0803.0883] [INSPIRE].

[26] P. Nason, A new method for combining NLO QCD with shower Monte Carlo algorithms, JHEP 11 (2004) 040 [hep-ph/0409146] [INSPIRE].

[27] S. Frixione, P. Nason and C. Oleari, Matching NLO QCD computations with Parton Shower simulations: the POWHEG method, JHEP 11 (2007) 070 [arXiv: 0709.2092] [INSPIRE].

[28] S. Alioli, P. Nason, C. Oleari and E. Re, A general framework for implementing NLO calculations in shower Monte Carlo programs: the POWHEG BOX, JHEP 06 (2010) 043 [arXiv: 1002.2581] [INSPIRE].

[29] S. Alioli, K. Hamilton, P. Nason, C. Oleari and E. Re, Jet pair production in POWHEG, JHEP 04 (2011) 081 [arXiv: 1012.3380] [InSPIRE].

[30] J. Alwall et al., The automated computation of tree-level and next-to-leading order differential cross sections and their matching to parton shower simulations, JHEP 07 (2014) 079 [arXiv: 1405.0301] [INSPIRE].

[31] Z. Nagy, Next-to-leading order calculation of three jet observables in hadron hadron collision, Phys. Rev. D 68 (2003) 094002 [hep-ph/0307268] [inSPIRE].

[32] S. Dulat et al., New parton distribution functions from a global analysis of quantum chromodynamics, Phys. Rev. D 93 (2016) 033006 [arXiv: 1506.07443] [INSPIRE].

[33] CMS collaboration, The CMS experiment at the CERN LHC, 2008 JINST 3 S08004 [INSPIRE]. 
[34] CMS collaboration, The CMS trigger system, 2017 JINST 12 P01020 [arXiv:1609.02366] [INSPIRE].

[35] CMS collaboration, Particle-flow reconstruction and global event description with the CMS detector, 2017 JINST 12 P10003 [arXiv:1706.04965] [INSPIRE].

[36] CMS collaboration, Pileup removal algorithms, CMS-PAS-JME-14-001.

[37] M. Cacciari, G.P. Salam and G. Soyez, The anti- $k_{t}$ jet clustering algorithm, JHEP 04 (2008) 063 [arXiv: 0802.1189] [INSPIRE].

[38] M. Cacciari, G.P. Salam and G. Soyez, FastJet user manual, Eur. Phys. J. C 72 (2012) 1896 [arXiv: 1111.6097] [INSPIRE].

[39] Y.L. Dokshitzer, G.D. Leder, S. Moretti and B.R. Webber, Better jet clustering algorithms, JHEP 08 (1997) 001 [hep-ph/9707323] [INSPIRE].

[40] M. Wobisch and T. Wengler, Hadronization corrections to jet cross-sections in deep inelastic scattering, in Monte Carlo generators for HERA physics. Proceedings, Workshop, Hamburg, Germany, 1998-1999, pp. 270-279 (1998) [hep-ph/9907280] [INSPIRE].

[41] M. Cacciari and G.P. Salam, Pileup subtraction using jet areas, Phys. Lett. B 659 (2008) 119 [arXiv: 0707.1378] [INSPIRE].

[42] CMS collaboration, Jet energy scale and resolution in the CMS experiment in pp collisions at $8 \mathrm{TeV}, 2017$ JINST 12 P02014 [arXiv:1607.03663] [INSPIRE].

[43] CMS collaboration, Search for massive resonances decaying into $W W, W Z$ or $Z Z$ bosons in proton-proton collisions at $\sqrt{s}=13 \mathrm{TeV}$, JHEP 03 (2017) 162 [arXiv: 1612.09159] [INSPIRE].

[44] CMS collaboration, Identification techniques for highly boosted $W$ bosons that decay into hadrons, JHEP 12 (2014) 017 [arXiv:1410.4227] [INSPIRE].

[45] CMS collaboration, Measurement of the double-differential inclusive jet cross section in proton-proton collisions at $\sqrt{s}=13 \mathrm{TeV}$, Eur. Phys. J. C 76 (2016) 451 [arXiv:1605.04436] [INSPIRE].

[46] CMS collaboration, Jet algorithms performance in 13 TeV data, CMS-PAS-JME-16-003.

[47] G. D'Agostini, A multidimensional unfolding method based on Bayes' theorem, Nucl. Instrum. Meth. A 362 (1995) 487 [INSPIRE].

[48] W.H. Richardson, Bayesian-based iterative method of image restoration, J. Opt. Soc. Am. 62 (1972) 55.

[49] L.B. Lucy, An iterative technique for the rectification of observed distributions, Astron. J. 79 (1974) 745 [INSPIRE].

[50] J. Tukey, J. Kiefer and B. Owen, Abstracts of papers, Ann. Math. Statis. 29 (1958) 614.

[51] M.H. Quenouille, Approximate tests of correlation in time-series, J. Royal Stat. Soc. B 11 (1949) 68 [http://www.jstor.org/stable/2983696].

[52] CMS collaboration, Determination of jet energy calibration and transverse momentum resolution in CMS, 2011 JINST 6 P11002 [arXiv:1107.4277] [INSPIRE].

[53] CMS collaboration, CMS luminosity measurement for the 2015 data-taking period, CMS-PAS-LUM-15-001. 


\section{The CMS collaboration}

\section{Yerevan Physics Institute, Yerevan, Armenia}

A.M. Sirunyan, A. Tumasyan

\section{Institut für Hochenergiephysik, Wien, Austria}

W. Adam, F. Ambrogi, E. Asilar, T. Bergauer, J. Brandstetter, M. Dragicevic, J. Erö, A. Escalante Del Valle, M. Flechl, R. Frühwirth ${ }^{1}$, V.M. Ghete, J. Hrubec, M. Jeitler ${ }^{1}$, N. Krammer, I. Krätschmer, D. Liko, T. Madlener, I. Mikulec, N. Rad, H. Rohringer, J. Schieck ${ }^{1}$, R. Schöfbeck, M. Spanring, D. Spitzbart, A. Taurok, W. Waltenberger, J. Wittmann, C.-E. Wulz ${ }^{1}$, M. Zarucki

\section{Institute for Nuclear Problems, Minsk, Belarus}

V. Chekhovsky, V. Mossolov, J. Suarez Gonzalez

Universiteit Antwerpen, Antwerpen, Belgium

E.A. De Wolf, D. Di Croce, X. Janssen, J. Lauwers, M. Pieters, H. Van Haevermaet, P. Van Mechelen, N. Van Remortel

\section{Vrije Universiteit Brussel, Brussel, Belgium}

S. Abu Zeid, F. Blekman, J. D'Hondt, I. De Bruyn, J. De Clercq, K. Deroover, G. Flouris, D. Lontkovskyi, S. Lowette, I. Marchesini, S. Moortgat, L. Moreels, Q. Python, K. Skovpen, S. Tavernier, W. Van Doninck, P. Van Mulders, I. Van Parijs

\section{Université Libre de Bruxelles, Bruxelles, Belgium}

D. Beghin, B. Bilin, H. Brun, B. Clerbaux, G. De Lentdecker, H. Delannoy, B. Dorney, G. Fasanella, L. Favart, R. Goldouzian, A. Grebenyuk, A.K. Kalsi, T. Lenzi, J. Luetic, N. Postiau, E. Starling, L. Thomas, C. Vander Velde, P. Vanlaer, D. Vannerom, Q. Wang

\section{Ghent University, Ghent, Belgium}

T. Cornelis, D. Dobur, A. Fagot, M. Gul, I. Khvastunov², D. Poyraz, C. Roskas, D. Trocino, M. Tytgat, W. Verbeke, B. Vermassen, M. Vit, N. Zaganidis

\section{Université Catholique de Louvain, Louvain-la-Neuve, Belgium}

H. Bakhshiansohi, O. Bondu, S. Brochet, G. Bruno, C. Caputo, P. David, C. Delaere, M. Delcourt, B. Francois, A. Giammanco, G. Krintiras, V. Lemaitre, A. Magitteri, A. Mertens, M. Musich, K. Piotrzkowski, A. Saggio, M. Vidal Marono, S. Wertz, J. Zobec

\section{Centro Brasileiro de Pesquisas Fisicas, Rio de Janeiro, Brazil}

F.L. Alves, G.A. Alves, M. Correa Martins Junior, G. Correia Silva, C. Hensel, A. Moraes, M.E. Pol, P. Rebello Teles

Universidade do Estado do Rio de Janeiro, Rio de Janeiro, Brazil

E. Belchior Batista Das Chagas, W. Carvalho, J. Chinellato ${ }^{3}$, E. Coelho, E.M. Da Costa, G.G. Da Silveira ${ }^{4}$, D. De Jesus Damiao, C. De Oliveira Martins, S. Fonseca De Souza, H. Malbouisson, D. Matos Figueiredo, M. Melo De Almeida, C. Mora Herrera, L. Mundim, H. Nogima, W.L. Prado Da Silva, L.J. Sanchez Rosas, A. Santoro, A. Sznajder, M. Thiel, E.J. Tonelli Manganote ${ }^{3}$, F. Torres Da Silva De Araujo, A. Vilela Pereira 
Universidade Estadual Paulista ${ }^{a}$, Universidade Federal do ABC ${ }^{b}$, São Paulo, Brazil

S. Ahuja ${ }^{a}$, C.A. Bernardes ${ }^{a}$, L. Calligaris $^{a}$, T.R. Fernandez Perez Tomei ${ }^{a}$, E.M. $_{\text {Gregores }}{ }^{b}$, P.G. Mercadante ${ }^{b}$, S.F. Novaes ${ }^{a}$, SandraS. Padula ${ }^{a}$

Institute for Nuclear Research and Nuclear Energy, Bulgarian Academy of Sciences, Sofia, Bulgaria

A. Aleksandrov, R. Hadjiiska, P. Iaydjiev, A. Marinov, M. Misheva, M. Rodozov,

M. Shopova, G. Sultanov

University of Sofia, Sofia, Bulgaria

A. Dimitrov, L. Litov, B. Pavlov, P. Petkov

Beihang University, Beijing, China

W. Fang ${ }^{5}$, X. Gao ${ }^{5}$, L. Yuan

Institute of High Energy Physics, Beijing, China

M. Ahmad, J.G. Bian, G.M. Chen, H.S. Chen, M. Chen, Y. Chen, C.H. Jiang, D. Leggat,

H. Liao, Z. Liu, F. Romeo, S.M. Shaheen ${ }^{6}$, A. Spiezia, J. Tao, C. Wang, Z. Wang, E. Yazgan,

H. Zhang, S. Zhang, J. Zhao

State Key Laboratory of Nuclear Physics and Technology, Peking University, Beijing, China

Y. Ban, G. Chen, A. Levin, J. Li, L. Li, Q. Li, Y. Mao, S.J. Qian, D. Wang, Z. Xu

Tsinghua University, Beijing, China

Y. Wang

Universidad de Los Andes, Bogota, Colombia

C. Avila, A. Cabrera, C.A. Carrillo Montoya, L.F. Chaparro Sierra, C. Florez,

C.F. González Hernández, M.A. Segura Delgado

University of Split, Faculty of Electrical Engineering, Mechanical Engineering and Naval Architecture, Split, Croatia

B. Courbon, N. Godinovic, D. Lelas, I. Puljak, T. Sculac

University of Split, Faculty of Science, Split, Croatia

Z. Antunovic, M. Kovac

Institute Rudjer Boskovic, Zagreb, Croatia

V. Brigljevic, D. Ferencek, K. Kadija, B. Mesic, A. Starodumov ${ }^{7}$, T. Susa

University of Cyprus, Nicosia, Cyprus

M.W. Ather, A. Attikis, M. Kolosova, G. Mavromanolakis, J. Mousa, C. Nicolaou, F. Ptochos, P.A. Razis, H. Rykaczewski

Charles University, Prague, Czech Republic

M. Finger ${ }^{8}$, M. Finger Jr. ${ }^{8}$ 
Escuela Politecnica Nacional, Quito, Ecuador

E. Ayala

Universidad San Francisco de Quito, Quito, Ecuador

E. Carrera Jarrin

Academy of Scientific Research and Technology of the Arab Republic of Egypt, Egyptian Network of High Energy Physics, Cairo, Egypt

A.A. Abdelalim ${ }^{9,10}$, A. Mahrous ${ }^{9}$, A. Mohamed $^{10}$

National Institute of Chemical Physics and Biophysics, Tallinn, Estonia

S. Bhowmik, A. Carvalho Antunes De Oliveira, R.K. Dewanjee, K. Ehataht, M. Kadastik, M. Raidal, C. Veelken

Department of Physics, University of Helsinki, Helsinki, Finland

P. Eerola, H. Kirschenmann, J. Pekkanen, M. Voutilainen

Helsinki Institute of Physics, Helsinki, Finland

J. Havukainen, J.K. Heikkilä, T. Järvinen, V. Karimäki, R. Kinnunen, T. Lampén, K. Lassila-Perini, S. Laurila, S. Lehti, T. Lindén, P. Luukka, T. Mäenpää, H. Siikonen, E. Tuominen, J. Tuominiemi

\section{Lappeenranta University of Technology, Lappeenranta, Finland}

T. Tuuva

IRFU, CEA, Université Paris-Saclay, Gif-sur-Yvette, France

M. Besancon, F. Couderc, M. Dejardin, D. Denegri, J.L. Faure, F. Ferri, S. Ganjour, A. Givernaud, P. Gras, G. Hamel de Monchenault, P. Jarry, C. Leloup, E. Locci, J. Malcles, G. Negro, J. Rander, A. Rosowsky, M.Ö. Sahin, M. Titov

Laboratoire Leprince-Ringuet, Ecole polytechnique, CNRS/IN2P3, Université Paris-Saclay, Palaiseau, France

A. Abdulsalam ${ }^{11}$, C. Amendola, I. Antropov, F. Beaudette, P. Busson, C. Charlot, R. Granier de Cassagnac, I. Kucher, A. Lobanov, J. Martin Blanco, M. Nguyen, C. Ochando, G. Ortona, P. Paganini, P. Pigard, R. Salerno, J.B. Sauvan, Y. Sirois, A.G. Stahl Leiton, A. Zabi, A. Zghiche

Université de Strasbourg, CNRS, IPHC UMR 7178, Strasbourg, France J.-L. Agram ${ }^{12}$, J. Andrea, D. Bloch, J.-M. Brom, E.C. Chabert, V. Cherepanov, C. Collard, E. Conte ${ }^{12}$, J.-C. Fontaine ${ }^{12}$, D. Gelé, U. Goerlach, M. Jansová, A.-C. Le Bihan, N. Tonon, P. Van Hove

Centre de Calcul de l'Institut National de Physique Nucleaire et de Physique des Particules, CNRS/IN2P3, Villeurbanne, France

S. Gadrat 
Université de Lyon, Université Claude Bernard Lyon 1, CNRS-IN2P3, Institut de Physique Nucléaire de Lyon, Villeurbanne, France

S. Beauceron, C. Bernet, G. Boudoul, N. Chanon, R. Chierici, D. Contardo, P. Depasse, H. El Mamouni, J. Fay, L. Finco, S. Gascon, M. Gouzevitch, G. Grenier, B. Ille, F. Lagarde, I.B. Laktineh, H. Lattaud, M. Lethuillier, L. Mirabito, A.L. Pequegnot, S. Perries, A. Popov ${ }^{13}$, V. Sordini, G. Touquet, M. Vander Donckt, S. Viret

Georgian Technical University, Tbilisi, Georgia

A. Khvedelidze ${ }^{8}$

Tbilisi State University, Tbilisi, Georgia

Z. Tsamalaidze ${ }^{8}$

RWTH Aachen University, I. Physikalisches Institut, Aachen, Germany

C. Autermann, L. Feld, M.K. Kiesel, K. Klein, M. Lipinski, M. Preuten, M.P. Rauch, C. Schomakers, J. Schulz, M. Teroerde, B. Wittmer, V. Zhukov ${ }^{13}$

RWTH Aachen University, III. Physikalisches Institut A, Aachen, Germany

A. Albert, D. Duchardt, M. Endres, M. Erdmann, S. Ghosh, A. Güth, T. Hebbeker, C. Heidemann, K. Hoepfner, H. Keller, L. Mastrolorenzo, M. Merschmeyer, A. Meyer, P. Millet, S. Mukherjee, T. Pook, M. Radziej, H. Reithler, M. Rieger, A. Schmidt, D. Teyssier

RWTH Aachen University, III. Physikalisches Institut B, Aachen, Germany

G. Flügge, O. Hlushchenko, T. Kress, A. Künsken, T. Müller, A. Nehrkorn, A. Nowack, C. Pistone, O. Pooth, D. Roy, H. Sert, A. Stahl ${ }^{14}$

\section{Deutsches Elektronen-Synchrotron, Hamburg, Germany}

M. Aldaya Martin, T. Arndt, C. Asawatangtrakuldee, I. Babounikau, K. Beernaert, O. Behnke, U. Behrens, A. Bermúdez Martínez, D. Bertsche, A.A. Bin Anuar, K. Borras ${ }^{15}$, V. Botta, A. Campbell, P. Connor, C. Contreras-Campana, F. Costanza, V. Danilov, A. De Wit, M.M. Defranchis, C. Diez Pardos, D. Domínguez Damiani, G. Eckerlin, T. Eichhorn, A. Elwood, E. Eren, E. Gallo ${ }^{16}$, A. Geiser, J.M. Grados Luyando, A. Grohsjean, P. Gunnellini, M. Guthoff, M. Haranko, A. Harb, J. Hauk, H. Jung, M. Kasemann, J. Keaveney, C. Kleinwort, J. Knolle, D. Krücker, W. Lange, A. Lelek, T. Lenz, K. Lipka, W. Lohmann ${ }^{17}$, R. Mankel, I.-A. Melzer-Pellmann, A.B. Meyer, M. Meyer, M. Missiroli, G. Mittag, J. Mnich, V. Myronenko, S.K. Pflitsch, D. Pitzl, A. Raspereza, M. Savitskyi, P. Saxena, P. Schütze, C. Schwanenberger, R. Shevchenko, A. Singh, H. Tholen, O. Turkot, A. Vagnerini, G.P. Van Onsem, R. Walsh, Y. Wen, K. Wichmann, C. Wissing, O. Zenaiev

\section{University of Hamburg, Hamburg, Germany}

R. Aggleton, S. Bein, L. Benato, A. Benecke, V. Blobel, M. Centis Vignali, T. Dreyer, E. Garutti, D. Gonzalez, J. Haller, A. Hinzmann, A. Karavdina, G. Kasieczka, R. Klanner, R. Kogler, N. Kovalchuk, S. Kurz, V. Kutzner, J. Lange, D. Marconi, J. Multhaup, M. Niedziela, D. Nowatschin, A. Perieanu, A. Reimers, O. Rieger, C. Scharf, P. Schleper, S. Schumann, J. Schwandt, J. Sonneveld, H. Stadie, G. Steinbrück, F.M. Stober, M. Stöver, D. Troendle, A. Vanhoefer, B. Vormwald 
Karlsruher Institut fuer Technology

M. Akbiyik, C. Barth, M. Baselga, S. Baur, E. Butz, R. Caspart, T. Chwalek, F. Colombo, W. De Boer, A. Dierlamm, K. El Morabit, N. Faltermann, B. Freund, M. Giffels, M.A. Harrendorf, F. Hartmann ${ }^{14}$, S.M. Heindl, U. Husemann, F. Kassel ${ }^{14}$, I. Katkov ${ }^{13}$, S. Kudella, H. Mildner, S. Mitra, M.U. Mozer, Th. Müller, M. Plagge, G. Quast, K. Rabbertz, M. Schröder, I. Shvetsov, G. Sieber, H.J. Simonis, R. Ulrich, S. Wayand, M. Weber, T. Weiler, S. Williamson, C. Wöhrmann, R. Wolf

Institute of Nuclear and Particle Physics (INPP), NCSR Demokritos, Aghia Paraskevi, Greece

G. Anagnostou, G. Daskalakis, T. Geralis, A. Kyriakis, D. Loukas, G. Paspalaki, I. TopsisGiotis

National and Kapodistrian University of Athens, Athens, Greece

G. Karathanasis, S. Kesisoglou, P. Kontaxakis, A. Panagiotou, I. Papavergou, N. Saoulidou, E. Tziaferi, K. Vellidis

National Technical University of Athens, Athens, Greece

K. Kousouris, I. Papakrivopoulos, G. Tsipolitis

University of Ioánnina, Ioánnina, Greece

I. Evangelou, C. Foudas, P. Gianneios, P. Katsoulis, P. Kokkas, S. Mallios, N. Manthos, I. Papadopoulos, E. Paradas, J. Strologas, F.A. Triantis, D. Tsitsonis

MTA-ELTE Lendület CMS Particle and Nuclear Physics Group, Eötvös Loránd University, Budapest, Hungary

M. Bartók ${ }^{18}$, M. Csanad, N. Filipovic, P. Major, M.I. Nagy, G. Pasztor, O. Surányi, G.I. Veres

Wigner Research Centre for Physics, Budapest, Hungary

G. Bencze, C. Hajdu, D. Horvath ${ }^{19}$, Á. Hunyadi, F. Sikler, T.Á. Vámi, V. Veszpremi, G. Vesztergombi ${ }^{\dagger}$

Institute of Nuclear Research ATOMKI, Debrecen, Hungary

N. Beni, S. Czellar, J. Karancsi ${ }^{20}$, A. Makovec, J. Molnar, Z. Szillasi

Institute of Physics, University of Debrecen, Debrecen, Hungary

P. Raics, Z.L. Trocsanyi, B. Ujvari

Indian Institute of Science (IISc), Bangalore, India

S. Choudhury, J.R. Komaragiri, P.C. Tiwari

National Institute of Science Education and Research, HBNI, Bhubaneswar, India

S. Bahinipati21 , C. Kar, P. Mal, K. Mandal, A. Nayak ${ }^{22}$, D.K. Sahoo ${ }^{21}$, S.K. Swain 
Panjab University, Chandigarh, India

S. Bansal, S.B. Beri, V. Bhatnagar, S. Chauhan, R. Chawla, N. Dhingra, R. Gupta, A. Kaur, M. Kaur, S. Kaur, R. Kumar, P. Kumari, M. Lohan, A. Mehta, K. Sandeep, S. Sharma, J.B. Singh, A.K. Virdi, G. Walia

\section{University of Delhi, Delhi, India}

A. Bhardwaj, B.C. Choudhary, R.B. Garg, M. Gola, S. Keshri, Ashok Kumar, S. Malhotra, M. Naimuddin, P. Priyanka, K. Ranjan, Aashaq Shah, R. Sharma

Saha Institute of Nuclear Physics, HBNI, Kolkata, India

R. Bhardwaj ${ }^{23}$, M. Bharti, R. Bhattacharya, S. Bhattacharya, U. Bhawandeep ${ }^{23}$, D. Bhowmik, S. Dey, S. Dutt ${ }^{23}$, S. Dutta, S. Ghosh, K. Mondal, S. Nandan, A. Purohit, P.K. Rout, A. Roy, S. Roy Chowdhury, G. Saha, S. Sarkar, M. Sharan, B. Singh, S. Thakur ${ }^{23}$

\section{Indian Institute of Technology Madras, Madras, India}

P.K. Behera

\section{Bhabha Atomic Research Centre, Mumbai, India}

R. Chudasama, D. Dutta, V. Jha, V. Kumar, P.K. Netrakanti, L.M. Pant, P. Shukla

Tata Institute of Fundamental Research-A, Mumbai, India

T. Aziz, M.A. Bhat, S. Dugad, G.B. Mohanty, N. Sur, B. Sutar, RavindraKumar Verma

Tata Institute of Fundamental Research-B, Mumbai, India

S. Banerjee, S. Bhattacharya, S. Chatterjee, P. Das, M. Guchait, Sa. Jain, S. Karmakar, S. Kumar, M. Maity ${ }^{24}$, G. Majumder, K. Mazumdar, N. Sahoo, T. Sarkar²4

Indian Institute of Science Education and Research (IISER), Pune, India

S. Chauhan, S. Dube, V. Hegde, A. Kapoor, K. Kothekar, S. Pandey, A. Rane, S. Sharma

\section{Institute for Research in Fundamental Sciences (IPM), Tehran, Iran}

S. Chenarani ${ }^{25}$, E. Eskandari Tadavani, S.M. Etesami ${ }^{25}$, M. Khakzad, M. Mohammadi Najafabadi, M. Naseri, F. Rezaei Hosseinabadi, B. Safarzadeh ${ }^{26}$, M. Zeinali

\section{University College Dublin, Dublin, Ireland}

M. Felcini, M. Grunewald

INFN Sezione di Bari ${ }^{a}$, Università di Bari ${ }^{b}$, Politecnico di Bari ${ }^{c}$, Bari, Italy M. Abbrescia ${ }^{a, b}$, C. Calabria ${ }^{a, b}$, A. Colaleo ${ }^{a}$, D. Creanza ${ }^{a, c}$, L. Cristella ${ }^{a, b}$, N. De Filippis ${ }^{a, c}$, M. De Palma ${ }^{a, b}$, A. Di Florio ${ }^{a, b}$, F. Errico ${ }^{a, b}$, L. Fiore ${ }^{a}$, A. Gelmi ${ }^{a, b}$, G. Iaselli ${ }^{a, c}$, M. Ince ${ }^{a, b}$, S. Lezki ${ }^{a, b}$, G. Maggi ${ }^{a, c}$, M. Maggi ${ }^{a}$, G. Miniello ${ }^{a, b}, \mathrm{~S}_{\text {. My }}{ }^{a, b}$, S. Nuzzo ${ }^{a, b}$, A. Pompilia, ${ }^{a, b}$ G. Pugliese ${ }^{a, c}$, R. Radogna ${ }^{a}$, A. Ranieri ${ }^{a}$, G. Selvaggi ${ }^{a}, b$, A. Sharma ${ }^{a}$, L. Silvestris ${ }^{a}$, R. Venditti ${ }^{a}$, P. Verwilligen ${ }^{a}$, G. Zito ${ }^{a}$

INFN Sezione di Bologna ${ }^{a}$, Università di Bologna ${ }^{b}$, Bologna, Italy

G. Abbiendi ${ }^{a}$, C. Battilana ${ }^{a, b}$, D. Bonacorsi ${ }^{a}, b$, L. Borgonovi $^{a, b}$, S. Braibant-Giacomelli ${ }^{a, b}$,

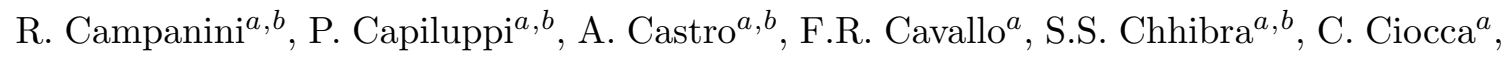

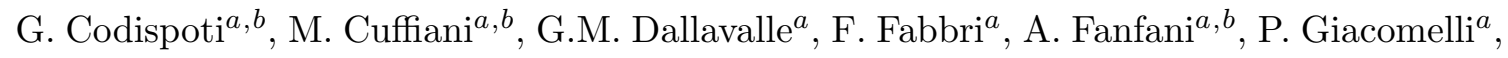


C. Grandi ${ }^{a}$, L. Guiducci ${ }^{a}, b$, F. Iemmi ${ }^{a}, b$, S. Marcellini ${ }^{a}$, G. Masetti ${ }^{a}$, A. Montanari ${ }^{a}$,

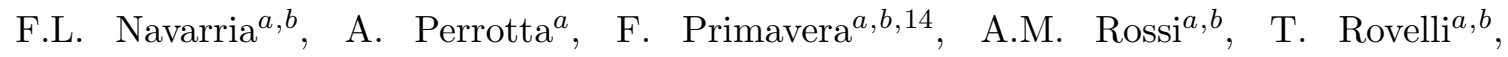
G.P. Siroli ${ }^{a, b}$, N. Tosi ${ }^{a}$

INFN Sezione di Catania ${ }^{a}$, Università di Catania ${ }^{b}$, Catania, Italy

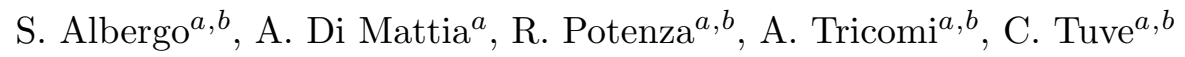

INFN Sezione di Firenze ${ }^{a}$, Università di Firenze ${ }^{b}$, Firenze, Italy

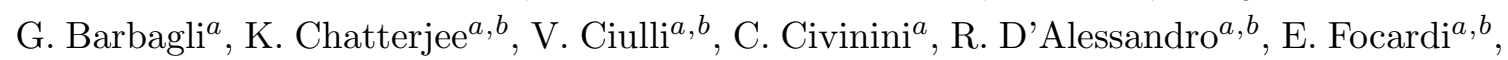

G. Latino, P. Lenzi ${ }^{a}, b$, M. Meschini ${ }^{a}$, S. Paoletti ${ }^{a}$, L. Russo $^{a, 27}$, G. Sguazzoni ${ }^{a}$, D. Strom ${ }^{a}$,

L. Viliani ${ }^{a}$

INFN Laboratori Nazionali di Frascati, Frascati, Italy

L. Benussi, S. Bianco, F. Fabbri, D. Piccolo

INFN Sezione di Genova ${ }^{a}$, Università di Genova ${ }^{b}$, Genova, Italy

F. Ferro $^{a}$, F. Ravera ${ }^{a, b}$, E. Robutti $^{a}$, S. Tosi ${ }^{a, b}$

INFN Sezione di Milano-Bicocca ${ }^{a}$, Università di Milano-Bicocca ${ }^{b}$, Milano, Italy

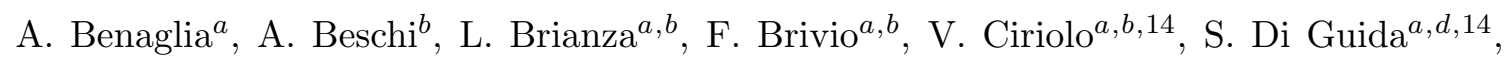
M.E. Dinardo ${ }^{a, b}$, S. Fiorendi ${ }^{a}, b$, S. Gennai ${ }^{a}$, A. Ghezzi ${ }^{a, b}$, P. Govoni $^{a, b}$, M. Malberti ${ }^{a, b}$, S. Malvezzi ${ }^{a}$, A. Massironi ${ }^{a, b}$, D. Menasce ${ }^{a}$, L. Moroni ${ }^{a}$, M. Paganoni $^{a}, b$, D. Pedrini ${ }^{a}$, S. Ragazzi ${ }^{a, b}$, T. Tabarelli de Fatis ${ }^{a, b}$, D. Zuolo

INFN Sezione di Napoli ${ }^{a}$, Università di Napoli 'Federico II' ${ }^{b}$, Napoli, Italy, Università della Basilicata ${ }^{c}$, Potenza, Italy, Università G. Marconi ${ }^{d}$, Roma, Italy

S. Buontempo ${ }^{a}$, N. Cavallo ${ }^{a, c}$, A. Di Crescenzo ${ }^{a, b}$, F. Fabozzi $^{a, c}$, F. Fienga $^{a}$, G. Galati $^{a}$, A.O.M. Iorio ${ }^{a, b}$, W.A. Khan ${ }^{a}$, L. Lista ${ }^{a}$, S. Meola ${ }^{a, d, 14}$, P. Paolucci ${ }^{a, 14}$, C. Sciacca ${ }^{a, b}$, E. Voevodina ${ }^{a, b}$

INFN Sezione di Padova ${ }^{a}$, Università di Padova ${ }^{b}$, Padova, Italy, Università di Trento ${ }^{c}$, Trento, Italy

P. Azzi ${ }^{a}$, N. Bacchetta ${ }^{a}$, D. Bisello ${ }^{a, b}$, A. Boletti ${ }^{a, b}$, A. Bragagnolo, R. Carlin ${ }^{a, b}$, P. Checchia ${ }^{a}$, M. Dall'Osso ${ }^{a, b}$, P. De Castro Manzano ${ }^{a}$, T. Dorigo ${ }^{a}$, U. Dosselli ${ }^{a}$, F. Gasparini ${ }^{a, b}$, U. Gasparini ${ }^{a, b}$, A. Gozzelino ${ }^{a}$, S.Y. Hoh, S. Lacaprara ${ }^{a}$, P. Lujan, M. Margoni ${ }^{a, b}$, A.T. Meneguzzo ${ }^{a, b}$, J. Pazzini ${ }^{a, b}$, N. Pozzobon ${ }^{a, b}$, P. Ronchese $^{a, b}$, R. Rossin ${ }^{a, b}$, F. Simonetto ${ }^{a, b}$, A. Tiko, E. Torassa ${ }^{a}$, M. Zanetti ${ }^{a, b}$, G. Zumerle ${ }^{a, b}$

INFN Sezione di Pavia ${ }^{a}$, Università di Pavia ${ }^{b}$, Pavia, Italy

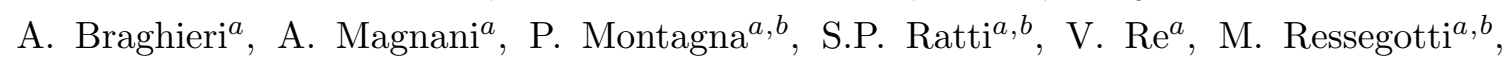
C. Riccardi ${ }^{a, b}$, P. Salvini ${ }^{a}$, I. Vai ${ }^{a}, b$, P. Vitulo ${ }^{a, b}$

INFN Sezione di Perugia ${ }^{a}$, Università di Perugia ${ }^{b}$, Perugia, Italy

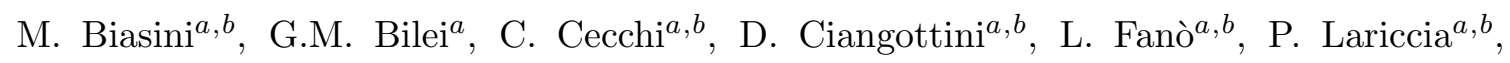

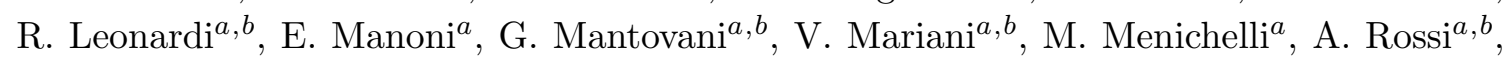
A. Santocchia ${ }^{a, b}$, D. Spiga ${ }^{a}$ 
INFN Sezione di Pisa ${ }^{a}$, Università di Pisa ${ }^{b}$, Scuola Normale Superiore di Pisa ${ }^{c}$, Pisa, Italy

K. Androsov ${ }^{a}$, P. Azzurri ${ }^{a}$, G. Bagliesi ${ }^{a}$, L. Bianchini ${ }^{a}$, T. Boccali ${ }^{a}$, L. Borrello, R. Castaldi ${ }^{a}$, M.A. Ciocci ${ }^{a}, b$, R. Dell'Orso ${ }^{a}$, G. Fedi ${ }^{a}$, F. Fiori ${ }^{a, c}$, L. Giannini $^{a, c}$, A. Giassi $^{a}$,

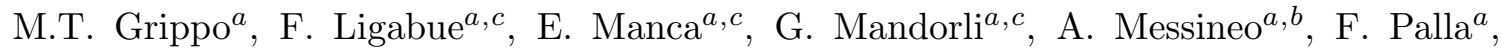

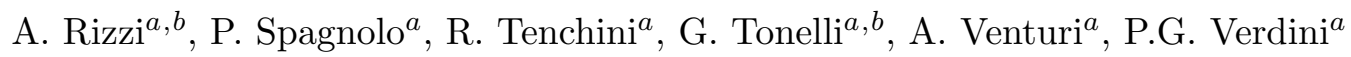

INFN Sezione di Roma ${ }^{a}$, Sapienza Università di Roma ${ }^{b}$, Rome, Italy

L. Barone ${ }^{a, b}$, F. Cavallari ${ }^{a}$, M. Cipriani ${ }^{a, b}$, N. Daci ${ }^{a}$, D. Del Re ${ }^{a, b}$, E. Di Marco ${ }^{a, b}$, M. Diemoz ${ }^{a}$, S. Gelli ${ }^{a, b}$, E. Longo ${ }^{a, b}$, B. Marzocchi ${ }^{a, b}$, P. Meridiani $^{a}$, G. Organtini ${ }^{a, b}$,

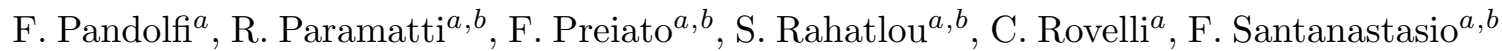

INFN Sezione di Torino ${ }^{a}$, Università di Torino ${ }^{b}$, Torino, Italy, Università del Piemonte Orientale ${ }^{c}$, Novara, Italy

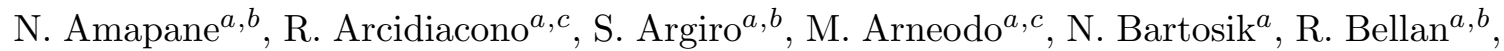

C. Biino ${ }^{a}$, N. Cartiglia ${ }^{a}$, F. Cenna ${ }^{a, b}$, S. Cometti ${ }^{a}$, M. Costa ${ }^{a, b}$, R. Covarelli ${ }^{a, b}$,

N. Demaria ${ }^{a}$, B. Kiani ${ }^{a, b}$, C. Mariotti ${ }^{a}$, S. Maselli ${ }^{a}$, E. Migliore ${ }^{a, b}$, V. Monaco ${ }^{a, b}$,

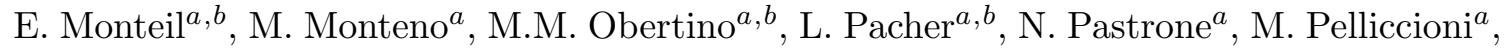
G.L. Pinna Angioni ${ }^{a, b}$, A. Romero ${ }^{a, b}$, M. Ruspa ${ }^{a, c}$, R. Sacchi ${ }^{a, b}$, K. Shchelina ${ }^{a, b}$, V. Sola ${ }^{a}$, A. Solano ${ }^{a, b}$, D. Soldi ${ }^{a, b}$, A. Staiano ${ }^{a}$

INFN Sezione di Trieste ${ }^{a}$, Università di Trieste ${ }^{b}$, Trieste, Italy

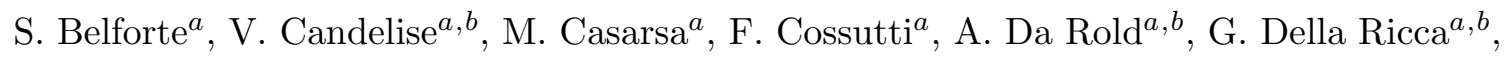
F. Vazzoler ${ }^{a, b}$, A. Zanetti ${ }^{a}$

\section{Kyungpook National University}

D.H. Kim, G.N. Kim, M.S. Kim, J. Lee, S. Lee, S.W. Lee, C.S. Moon, Y.D. Oh, S. Sekmen, D.C. Son, Y.C. Yang

Chonnam National University, Institute for Universe and Elementary Particles, Kwangju, Korea

H. Kim, D.H. Moon, G. Oh

Hanyang University, Seoul, Korea

J. Goh ${ }^{28}$, T.J. Kim

Korea University, Seoul, Korea

S. Cho, S. Choi, Y. Go, D. Gyun, S. Ha, B. Hong, Y. Jo, K. Lee, K.S. Lee, S. Lee, J. Lim, S.K. Park, Y. Roh

Sejong University, Seoul, Korea

H.S. Kim

Seoul National University, Seoul, Korea

J. Almond, J. Kim, J.S. Kim, H. Lee, K. Lee, K. Nam, S.B. Oh, B.C. Radburn-Smith, S.h. Seo, U.K. Yang, H.D. Yoo, G.B. Yu

University of Seoul, Seoul, Korea

D. Jeon, H. Kim, J.H. Kim, J.S.H. Lee, I.C. Park 
Sungkyunkwan University, Suwon, Korea

Y. Choi, C. Hwang, J. Lee, I. Yu

Vilnius University, Vilnius, Lithuania

V. Dudenas, A. Juodagalvis, J. Vaitkus

National Centre for Particle Physics, Universiti Malaya, Kuala Lumpur, Malaysia

I. Ahmed, Z.A. Ibrahim, M.A.B. Md Ali ${ }^{29}$, F. Mohamad Idris ${ }^{30}$, W.A.T. Wan Abdullah, M.N. Yusli, Z. Zolkapli

Universidad de Sonora (UNISON), Hermosillo, Mexico

J.F. Benitez, A. Castaneda Hernandez, J.A. Murillo Quijada

Centro de Investigacion y de Estudios Avanzados del IPN, Mexico City, Mexico

H. Castilla-Valdez, E. De La Cruz-Burelo, M.C. Duran-Osuna, I. Heredia-De La Cruz ${ }^{31}$,

R. Lopez-Fernandez, J. Mejia Guisao, R.I. Rabadan-Trejo, M. Ramirez-Garcia,

G. Ramirez-Sanchez, R Reyes-Almanza, A. Sanchez-Hernandez

Universidad Iberoamericana, Mexico City, Mexico

S. Carrillo Moreno, C. Oropeza Barrera, F. Vazquez Valencia

Benemerita Universidad Autonoma de Puebla, Puebla, Mexico

J. Eysermans, I. Pedraza, H.A. Salazar Ibarguen, C. Uribe Estrada

Universidad Autónoma de San Luis Potosí, San Luis Potosí, Mexico

A. Morelos Pineda

University of Auckland, Auckland, New Zealand

D. Krofcheck

University of Canterbury, Christchurch, New Zealand

S. Bheesette, P.H. Butler

National Centre for Physics, Quaid-I-Azam University, Islamabad, Pakistan

A. Ahmad, M. Ahmad, M.I. Asghar, Q. Hassan, H.R. Hoorani, A. Saddique, M.A. Shah, M. Shoaib, M. Waqas

National Centre for Nuclear Research, Swierk, Poland

H. Bialkowska, M. Bluj, B. Boimska, T. Frueboes, M. Górski, M. Kazana, K. Nawrocki, M. Szleper, P. Traczyk, P. Zalewski

Institute of Experimental Physics, Faculty of Physics, University of Warsaw, Warsaw, Poland

K. Bunkowski, A. Byszuk ${ }^{32}$, K. Doroba, A. Kalinowski, M. Konecki, J. Krolikowski, M. Misiura, M. Olszewski, A. Pyskir, M. Walczak 
Laboratório de Instrumentação e Física Experimental de Partículas, Lisboa, Portugal

M. Araujo, P. Bargassa, C. Beirão Da Cruz E Silva, A. Di Francesco, P. Faccioli, B. Galinhas, M. Gallinaro, J. Hollar, N. Leonardo, M.V. Nemallapudi, J. Seixas, G. Strong, O. Toldaiev, D. Vadruccio, J. Varela

\section{Joint Institute for Nuclear Research, Dubna, Russia}

S. Afanasiev, P. Bunin, M. Gavrilenko, I. Golutvin, I. Gorbunov, A. Kamenev, V. Karjavine, A. Lanev, A. Malakhov, V. Matveev ${ }^{33,34}$, P. Moisenz, V. Palichik, V. Perelygin, S. Shmatov, S. Shulha, N. Skatchkov, V. Smirnov, N. Voytishin, A. Zarubin

Petersburg Nuclear Physics Institute, Gatchina (St. Petersburg), Russia

V. Golovtsov, Y. Ivanov, V. Kim ${ }^{35}$, E. Kuznetsova ${ }^{36}$, P. Levchenko, V. Murzin, V. Oreshkin, I. Smirnov, D. Sosnov, V. Sulimov, L. Uvarov, S. Vavilov, A. Vorobyev

Institute for Nuclear Research, Moscow, Russia

Yu. Andreev, A. Dermenev, S. Gninenko, N. Golubev, A. Karneyeu, M. Kirsanov, N. Krasnikov, A. Pashenkov, D. Tlisov, A. Toropin

Institute for Theoretical and Experimental Physics, Moscow, Russia

V. Epshteyn, V. Gavrilov, N. Lychkovskaya, V. Popov, I. Pozdnyakov, G. Safronov, A. Spiridonov, A. Stepennov, V. Stolin, M. Toms, E. Vlasov, A. Zhokin

Moscow Institute of Physics and Technology, Moscow, Russia

T. Aushev

National Research Nuclear University 'Moscow Engineering Physics Institute' (MEPhI), Moscow, Russia

M. Chadeeva ${ }^{37}$, P. Parygin, D. Philippov, S. Polikarpov ${ }^{37}$, E. Popova, V. Rusinov

P.N. Lebedev Physical Institute, Moscow, Russia

V. Andreev, M. Azarkin ${ }^{34}$, I. Dremin ${ }^{34}$, M. Kirakosyan ${ }^{34}$, S.V. Rusakov, A. Terkulov

Skobeltsyn Institute of Nuclear Physics, Lomonosov Moscow State University, Moscow, Russia

A. Baskakov, A. Belyaev, E. Boos, M. Dubinin ${ }^{38}$, L. Dudko, A. Ershov, A. Gribushin, V. Klyukhin, O. Kodolova, I. Lokhtin, I. Miagkov, S. Obraztsov, S. Petrushanko, V. Savrin, A. Snigirev

Novosibirsk State University (NSU), Novosibirsk, Russia

A. Barnyakov ${ }^{39}$, V. Blinov ${ }^{39}$, T. Dimova ${ }^{39}$, L. Kardapoltsev ${ }^{39}$, Y. Skovpen ${ }^{39}$

State Research Center of Russian Federation, Institute for High Energy Physics of NRC "Kurchatov Institute", Protvino, Russia

I. Azhgirey, I. Bayshev, S. Bitioukov, D. Elumakhov, A. Godizov, V. Kachanov, A. Kalinin,

D. Konstantinov, P. Mandrik, V. Petrov, R. Ryutin, S. Slabospitskii, A. Sobol, S. Troshin, N. Tyurin, A. Uzunian, A. Volkov 
National Research Tomsk Polytechnic University, Tomsk, Russia

A. Babaev, S. Baidali, V. Okhotnikov

University of Belgrade, Faculty of Physics and Vinca Institute of Nuclear Sciences, Belgrade, Serbia

P. Adzic ${ }^{40}$, P. Cirkovic, D. Devetak, M. Dordevic, J. Milosevic

Centro de Investigaciones Energéticas Medioambientales y Tecnológicas (CIEMAT), Madrid, Spain

J. Alcaraz Maestre, A. Álvarez Fernández, I. Bachiller, M. Barrio Luna, J.A. Brochero Cifuentes, M. Cerrada, N. Colino, B. De La Cruz, A. Delgado Peris, C. Fernandez Bedoya, J.P. Fernández Ramos, J. Flix, M.C. Fouz, O. Gonzalez Lopez, S. Goy Lopez, J.M. Hernandez, M.I. Josa, D. Moran, A. Pérez-Calero Yzquierdo, J. Puerta Pelayo, I. Redondo, L. Romero, M.S. Soares, A. Triossi

Universidad Autónoma de Madrid, Madrid, Spain

C. Albajar, J.F. de Trocóniz

Universidad de Oviedo, Oviedo, Spain

J. Cuevas, C. Erice, J. Fernandez Menendez, S. Folgueras, I. Gonzalez Caballero, J.R. González Fernández, E. Palencia Cortezon, V. Rodríguez Bouza, S. Sanchez Cruz, P. Vischia, J.M. Vizan Garcia

Instituto de Física de Cantabria (IFCA), CSIC-Universidad de Cantabria, Santander, Spain

I.J. Cabrillo, A. Calderon, B. Chazin Quero, J. Duarte Campderros, M. Fernandez, P.J. Fernández Manteca, A. García Alonso, J. Garcia-Ferrero, G. Gomez, A. Lopez Virto, J. Marco, C. Martinez Rivero, P. Martinez Ruiz del Arbol, F. Matorras, J. Piedra Gomez, C. Prieels, T. Rodrigo, A. Ruiz-Jimeno, L. Scodellaro, N. Trevisani, I. Vila, R. Vilar Cortabitarte

University of Ruhuna, Department of Physics, Matara, Sri Lanka

N. Wickramage

\section{CERN, European Organization for Nuclear Research, Geneva, Switzerland}

D. Abbaneo, B. Akgun, E. Auffray, G. Auzinger, P. Baillon, A.H. Ball, D. Barney, J. Bendavid, M. Bianco, A. Bocci, C. Botta, E. Brondolin, T. Camporesi, M. Cepeda, G. Cerminara, E. Chapon, Y. Chen, G. Cucciati, D. d'Enterria, A. Dabrowski, V. Daponte, A. David, A. De Roeck, N. Deelen, M. Dobson, M. Dünser, N. Dupont, A. ElliottPeisert, P. Everaerts, F. Fallavollita ${ }^{41}$, D. Fasanella, G. Franzoni, J. Fulcher, W. Funk, D. Gigi, A. Gilbert, K. Gill, F. Glege, M. Guilbaud, D. Gulhan, J. Hegeman, C. Heidegger, V. Innocente, A. Jafari, P. Janot, O. Karacheban ${ }^{17}$, J. Kieseler, A. Kornmayer, M. Krammer ${ }^{1}$, C. Lange, P. Lecoq, C. Lourenço, L. Malgeri, M. Mannelli, F. Meijers, J.A. Merlin, S. Mersi, E. Meschi, P. Milenovic ${ }^{42}$, F. Moortgat, M. Mulders, J. Ngadiuba, S. Nourbakhsh, S. Orfanelli, L. Orsini, F. Pantaleo ${ }^{14}$, L. Pape, E. Perez, M. Peruzzi, A. Petrilli, G. Petrucciani, A. Pfeiffer, M. Pierini, F.M. Pitters, D. Rabady, A. Racz, 
T. Reis, G. Rolandi ${ }^{43}$, M. Rovere, H. Sakulin, C. Schäfer, C. Schwick, M. Seidel, M. Selvaggi, A. Sharma, P. Silva, P. Sphicas ${ }^{44}$, A. Stakia, J. Steggemann, M. Tosi, D. Treille, A. Tsirou, V. Veckalns ${ }^{45}$, M. Verzetti, W.D. Zeuner

Paul Scherrer Institut, Villigen, Switzerland

L. Caminada ${ }^{46}$, K. Deiters, W. Erdmann, R. Horisberger, Q. Ingram, H.C. Kaestli, D. Kotlinski, U. Langenegger, T. Rohe, S.A. Wiederkehr

ETH Zurich - Institute for Particle Physics and Astrophysics (IPA), Zurich, Switzerland

M. Backhaus, L. Bäni, P. Berger, N. Chernyavskaya, G. Dissertori, M. Dittmar, M. Donegà, C. Dorfer, C. Grab, D. Hits, J. Hoss, T. Klijnsma, W. Lustermann, R.A. Manzoni, M. Marionneau, M.T. Meinhard, F. Micheli, P. Musella, F. Nessi-Tedaldi, J. Pata, F. Pauss, G. Perrin, L. Perrozzi, S. Pigazzini, M. Quittnat, D. Ruini, D.A. Sanz Becerra, M. Schönenberger, L. Shchutska, V.R. Tavolaro, K. Theofilatos, M.L. Vesterbacka Olsson, R. Wallny, D.H. Zhu

\section{Universität Zürich, Zurich, Switzerland}

T.K. Aarrestad, C. Amsler ${ }^{47}$, D. Brzhechko, M.F. Canelli, A. De Cosa, R. Del Burgo, S. Donato, C. Galloni, T. Hreus, B. Kilminster, S. Leontsinis, I. Neutelings, D. Pinna, G. Rauco, P. Robmann, D. Salerno, K. Schweiger, C. Seitz, Y. Takahashi, A. Zucchetta

National Central University, Chung-Li, Taiwan

Y.H. Chang, K.y. Cheng, T.H. Doan, Sh. Jain, R. Khurana, C.M. Kuo, W. Lin, A. Pozdnyakov, S.S. Yu

National Taiwan University (NTU), Taipei, Taiwan

P. Chang, Y. Chao, K.F. Chen, P.H. Chen, W.-S. Hou, Arun Kumar, Y.y. Li, Y.F. Liu, R.-S. Lu, E. Paganis, A. Psallidas, A. Steen

Chulalongkorn University, Faculty of Science, Department of Physics, Bangkok, Thailand

B. Asavapibhop, N. Srimanobhas, N. Suwonjandee

Çukurova University, Physics Department, Science and Art Faculty, Adana, Turkey

M.N. Bakirci ${ }^{48}$, A. Bat, F. Boran, S. Cerci ${ }^{49}$, S. Damarseckin, Z.S. Demiroglu, F. Dolek, C. Dozen, E. Eskut, S. Girgis, G. Gokbulut, Y. Guler, E. Gurpinar, I. Hos ${ }^{50}$, C. Isik, E.E. Kangal ${ }^{51}$, O. Kara, U. Kiminsu, M. Oglakci, G. Onengut, K. Ozdemir ${ }^{52}$, A. Polatoz, D. Sunar Cerci $^{49}$, U.G. Tok, H. Topakli ${ }^{48}$, S. Turkcapar, I.S. Zorbakir, C. Zorbilmez

Middle East Technical University, Physics Department, Ankara, Turkey

B. Isildak ${ }^{53}$, G. Karapinar ${ }^{54}$, M. Yalvac, M. Zeyrek

Bogazici University, Istanbul, Turkey

I.O. Atakisi, E. Gülmez, M. Kaya ${ }^{55}$, O. Kaya ${ }^{56}$, S. Ozkorucuklu ${ }^{57}$, S. Tekten, E.A. Yetkin ${ }^{58}$

Istanbul Technical University, Istanbul, Turkey

M.N. Agaras, S. Atay, A. Cakir, K. Cankocak, Y. Komurcu, S. Sen ${ }^{59}$ 
Institute for Scintillation Materials of National Academy of Science of Ukraine, Kharkov, Ukraine

B. Grynyov

National Scientific Center, Kharkov Institute of Physics and Technology, Kharkov, Ukraine

L. Levchuk

University of Bristol, Bristol, United Kingdom

F. Ball, L. Beck, J.J. Brooke, D. Burns, E. Clement, D. Cussans, O. Davignon, H. Flacher, J. Goldstein, G.P. Heath, H.F. Heath, L. Kreczko, D.M. Newbold ${ }^{60}$, S. Paramesvaran, B. Penning, T. Sakuma, D. Smith, V.J. Smith, J. Taylor, A. Titterton

\section{Rutherford Appleton Laboratory, Didcot, United Kingdom}

K.W. Bell, A. Belyaev ${ }^{61}$, C. Brew, R.M. Brown, D. Cieri, D.J.A. Cockerill, J.A. Coughlan, K. Harder, S. Harper, J. Linacre, E. Olaiya, D. Petyt, C.H. Shepherd-Themistocleous, A. Thea, I.R. Tomalin, T. Williams, W.J. Womersley

\section{Imperial College, London, United Kingdom}

R. Bainbridge, P. Bloch, J. Borg, S. Breeze, O. Buchmuller, A. Bundock, S. Casasso,

D. Colling, L. Corpe, P. Dauncey, G. Davies, M. Della Negra, R. Di Maria, Y. Haddad, G. Hall, G. Iles, T. James, M. Komm, C. Laner, L. Lyons, A.-M. Magnan, S. Malik, A. Martelli, J. Nash ${ }^{62}$, A. Nikitenko ${ }^{7}$, V. Palladino, M. Pesaresi, A. Richards, A. Rose, E. Scott, C. Seez, A. Shtipliyski, G. Singh, M. Stoye, T. Strebler, S. Summers, A. Tapper, K. Uchida, T. Virdee ${ }^{14}$, N. Wardle, D. Winterbottom, J. Wright, S.C. Zenz

Brunel University, Uxbridge, United Kingdom

J.E. Cole, P.R. Hobson, A. Khan, P. Kyberd, C.K. Mackay, A. Morton, I.D. Reid, L. Teodorescu, S. Zahid

Baylor University, Waco, U.S.A.

K. Call, J. Dittmann, K. Hatakeyama, H. Liu, C. Madrid, B. Mcmaster, N. Pastika, C. Smith

Catholic University of America, Washington DC, U.S.A.

R. Bartek, A. Dominguez

The University of Alabama, Tuscaloosa, U.S.A.

A. Buccilli, S.I. Cooper, C. Henderson, P. Rumerio, C. West

Boston University, Boston, U.S.A.

D. Arcaro, T. Bose, D. Gastler, D. Rankin, C. Richardson, J. Rohlf, L. Sulak, D. Zou

Brown University, Providence, U.S.A.

G. Benelli, X. Coubez, D. Cutts, M. Hadley, J. Hakala, U. Heintz, J.M. Hogan ${ }^{63}$, K.H.M. Kwok, E. Laird, G. Landsberg, J. Lee, Z. Mao, M. Narain, S. Piperov, S. Sagir ${ }^{64}$, R. Syarif, E. Usai, D. Yu 
University of California, Davis, Davis, U.S.A.

R. Band, C. Brainerd, R. Breedon, D. Burns, M. Calderon De La Barca Sanchez, M. Chertok, J. Conway, R. Conway, P.T. Cox, R. Erbacher, C. Flores, G. Funk, W. Ko, O. Kukral, R. Lander, M. Mulhearn, D. Pellett, J. Pilot, S. Shalhout, M. Shi, D. Stolp, D. Taylor, K. Tos, M. Tripathi, Z. Wang, F. Zhang

University of California, Los Angeles, U.S.A.

M. Bachtis, C. Bravo, R. Cousins, A. Dasgupta, A. Florent, J. Hauser, M. Ignatenko, N. Mccoll, S. Regnard, D. Saltzberg, C. Schnaible, V. Valuev

University of California, Riverside, Riverside, U.S.A.

E. Bouvier, K. Burt, R. Clare, J.W. Gary, S.M.A. Ghiasi Shirazi, G. Hanson, G. Karapostoli, E. Kennedy, F. Lacroix, O.R. Long, M. Olmedo Negrete, M.I. Paneva, W. Si, L. Wang, H. Wei, S. Wimpenny, B.R. Yates

University of California, San Diego, La Jolla, U.S.A.

J.G. Branson, S. Cittolin, M. Derdzinski, R. Gerosa, D. Gilbert, B. Hashemi, A. Holzner, D. Klein, G. Kole, V. Krutelyov, J. Letts, M. Masciovecchio, D. Olivito, S. Padhi, M. Pieri, M. Sani, V. Sharma, S. Simon, M. Tadel, A. Vartak, S. Wasserbaech ${ }^{65}$, J. Wood, F. Würthwein, A. Yagil, G. Zevi Della Porta

University of California, Santa Barbara - Department of Physics, Santa Barbara, U.S.A.

N. Amin, R. Bhandari, J. Bradmiller-Feld, C. Campagnari, M. Citron, A. Dishaw, V. Dutta, M. Franco Sevilla, L. Gouskos, R. Heller, J. Incandela, A. Ovcharova, H. Qu, J. Richman, D. Stuart, I. Suarez, S. Wang, J. Yoo

California Institute of Technology, Pasadena, U.S.A.

D. Anderson, A. Bornheim, J.M. Lawhorn, H.B. Newman, T.Q. Nguyen, M. Spiropulu, J.R. Vlimant, R. Wilkinson, S. Xie, Z. Zhang, R.Y. Zhu

Carnegie Mellon University, Pittsburgh, U.S.A.

M.B. Andrews, T. Ferguson, T. Mudholkar, M. Paulini, M. Sun, I. Vorobiev, M. Weinberg University of Colorado Boulder, Boulder, U.S.A.

J.P. Cumalat, W.T. Ford, F. Jensen, A. Johnson, M. Krohn, E. MacDonald, T. Mulholland, R. Patel, K. Stenson, K.A. Ulmer, S.R. Wagner

Cornell University, Ithaca, U.S.A.

J. Alexander, J. Chaves, Y. Cheng, J. Chu, A. Datta, K. Mcdermott, N. Mirman, J.R. Patterson, D. Quach, A. Rinkevicius, A. Ryd, L. Skinnari, L. Soffi, S.M. Tan, Z. Tao, J. Thom, J. Tucker, P. Wittich, M. Zientek

Fermi National Accelerator Laboratory, Batavia, U.S.A.

S. Abdullin, M. Albrow, M. Alyari, G. Apollinari, A. Apresyan, A. Apyan, S. Banerjee, L.A.T. Bauerdick, A. Beretvas, J. Berryhill, P.C. Bhat, G. Bolla ${ }^{\dagger}$, K. Burkett, J.N. Butler, A. Canepa, G.B. Cerati, H.W.K. Cheung, F. Chlebana, M. Cremonesi, J. Duarte, V.D. Elvira, J. Freeman, Z. Gecse, E. Gottschalk, L. Gray, D. Green, S. Grünendahl, 
O. Gutsche, J. Hanlon, R.M. Harris, S. Hasegawa, J. Hirschauer, Z. Hu, B. Jayatilaka, S. Jindariani, M. Johnson, U. Joshi, B. Klima, M.J. Kortelainen, B. Kreis, S. Lammel, D. Lincoln, R. Lipton, M. Liu, T. Liu, J. Lykken, K. Maeshima, J.M. Marraffino, D. Mason, P. McBride, P. Merkel, S. Mrenna, S. Nahn, V. O’Dell, K. Pedro, C. Pena, O. Prokofyev, G. Rakness, L. Ristori, A. Savoy-Navarro ${ }^{66}$, B. Schneider, E. Sexton-Kennedy, A. Soha, W.J. Spalding, L. Spiegel, S. Stoynev, J. Strait, N. Strobbe, L. Taylor, S. Tkaczyk, N.V. Tran, L. Uplegger, E.W. Vaandering, C. Vernieri, M. Verzocchi, R. Vidal, M. Wang, H.A. Weber, A. Whitbeck

University of Florida, Gainesville, U.S.A.

D. Acosta, P. Avery, P. Bortignon, D. Bourilkov, A. Brinkerhoff, L. Cadamuro, A. Carnes, M. Carver, D. Curry, R.D. Field, S.V. Gleyzer, B.M. Joshi, J. Konigsberg, A. Korytov, P. Ma, K. Matchev, H. Mei, G. Mitselmakher, K. Shi, D. Sperka, J. Wang, S. Wang

Florida International University, Miami, U.S.A.

Y.R. Joshi, S. Linn

Florida State University, Tallahassee, U.S.A.

A. Ackert, T. Adams, A. Askew, S. Hagopian, V. Hagopian, K.F. Johnson, T. Kolberg, G. Martinez, T. Perry, H. Prosper, A. Saha, C. Schiber, V. Sharma, R. Yohay

Florida Institute of Technology, Melbourne, U.S.A.

M.M. Baarmand, V. Bhopatkar, S. Colafranceschi, M. Hohlmann, D. Noonan, M. Rahmani, T. Roy, F. Yumiceva

\section{University of Illinois at Chicago (UIC), Chicago, U.S.A.}

M.R. Adams, L. Apanasevich, D. Berry, R.R. Betts, R. Cavanaugh, X. Chen, S. Dittmer, O. Evdokimov, C.E. Gerber, D.A. Hangal, D.J. Hofman, K. Jung, J. Kamin, C. Mills, I.D. Sandoval Gonzalez, M.B. Tonjes, N. Varelas, H. Wang, X. Wang, Z. Wu, J. Zhang

The University of Iowa, Iowa City, U.S.A.

M. Alhusseini, B. Bilki ${ }^{67}$, W. Clarida, K. Dilsiz ${ }^{68}$, S. Durgut, R.P. Gandrajula, M. Haytmyradov, V. Khristenko, J.-P. Merlo, A. Mestvirishvili, A. Moeller, J. Nachtman, H. Ogul ${ }^{69}$, Y. Onel, F. Ozok ${ }^{70}$, A. Penzo, C. Snyder, E. Tiras, J. Wetzel

Johns Hopkins University, Baltimore, U.S.A.

B. Blumenfeld, A. Cocoros, N. Eminizer, D. Fehling, L. Feng, A.V. Gritsan, W.T. Hung, P. Maksimovic, J. Roskes, U. Sarica, M. Swartz, M. Xiao, C. You

The University of Kansas, Lawrence, U.S.A.

A. Al-bataineh, P. Baringer, A. Bean, S. Boren, J. Bowen, A. Bylinkin, J. Castle, S. Khalil, A. Kropivnitskaya, D. Majumder, W. Mcbrayer, M. Murray, C. Rogan, S. Sanders, E. Schmitz, J.D. Tapia Takaki, Q. Wang

Kansas State University, Manhattan, U.S.A.

S. Duric, A. Ivanov, K. Kaadze, D. Kim, Y. Maravin, D.R. Mendis, T. Mitchell, A. Modak, A. Mohammadi, L.K. Saini, N. Skhirtladze 
Lawrence Livermore National Laboratory, Livermore, U.S.A.

F. Rebassoo, D. Wright

University of Maryland, College Park, U.S.A.

A. Baden, O. Baron, A. Belloni, S.C. Eno, Y. Feng, C. Ferraioli, N.J. Hadley, S. Jabeen, G.Y. Jeng, R.G. Kellogg, J. Kunkle, A.C. Mignerey, F. Ricci-Tam, Y.H. Shin, A. Skuja, S.C. Tonwar, K. Wong

Massachusetts Institute of Technology, Cambridge, U.S.A.

D. Abercrombie, B. Allen, V. Azzolini, A. Baty, G. Bauer, R. Bi, S. Brandt, W. Busza, I.A. Cali, M. D'Alfonso, Z. Demiragli, G. Gomez Ceballos, M. Goncharov, P. Harris, D. Hsu, M. Hu, Y. Iiyama, G.M. Innocenti, M. Klute, D. Kovalskyi, Y.-J. Lee, P.D. Luckey, B. Maier, A.C. Marini, C. Mcginn, C. Mironov, S. Narayanan, X. Niu, C. Paus, C. Roland, G. Roland, G.S.F. Stephans, K. Sumorok, K. Tatar, D. Velicanu, J. Wang, T.W. Wang, B. Wyslouch, S. Zhaozhong

University of Minnesota, Minneapolis, U.S.A.

A.C. Benvenuti, R.M. Chatterjee, A. Evans, P. Hansen, S. Kalafut, Y. Kubota, Z. Lesko, J. Mans, N. Ruckstuhl, R. Rusack, J. Turkewitz, M.A. Wadud

University of Mississippi, Oxford, U.S.A.

J.G. Acosta, S. Oliveros

University of Nebraska-Lincoln, Lincoln, U.S.A.

E. Avdeeva, K. Bloom, D.R. Claes, C. Fangmeier, F. Golf, R. Gonzalez Suarez, R. Kamalieddin, I. Kravchenko, J. Monroy, J.E. Siado, G.R. Snow, B. Stieger

State University of New York at Buffalo, Buffalo, U.S.A.

A. Fortman, A. Godshalk, C. Harrington, I. Iashvili, A. Kharchilava, C. Mclean, D. Nguyen, A. Parker, J. Pusztay, S. Rappoccio, B. Roozbahani

Northeastern University, Boston, U.S.A.

E. Barberis, C. Freer, A. Hortiangtham, D.M. Morse, T. Orimoto, R. Teixeira De Lima, T. Wamorkar, B. Wang, A. Wisecarver, D. Wood

Northwestern University, Evanston, U.S.A.

S. Bhattacharya, O. Charaf, K.A. Hahn, N. Mucia, N. Odell, M.H. Schmitt, K. Sung, M. Trovato, M. Velasco

University of Notre Dame, Notre Dame, U.S.A.

R. Bucci, N. Dev, M. Hildreth, K. Hurtado Anampa, C. Jessop, D.J. Karmgard, N. Kellams, K. Lannon, W. Li, N. Loukas, N. Marinelli, F. Meng, C. Mueller, Y. Musienko ${ }^{33}$, M. Planer, A. Reinsvold, R. Ruchti, P. Siddireddy, G. Smith, S. Taroni, M. Wayne, A. Wightman, M. Wolf, A. Woodard

The Ohio State University, Columbus, U.S.A.

J. Alimena, L. Antonelli, B. Bylsma, L.S. Durkin, S. Flowers, B. Francis, A. Hart, C. Hill, W. Ji, T.Y. Ling, W. Luo, B.L. Winer, H.W. Wulsin 
Princeton University, Princeton, U.S.A.

S. Cooperstein, P. Elmer, J. Hardenbrook, S. Higginbotham, A. Kalogeropoulos, D. Lange, M.T. Lucchini, J. Luo, D. Marlow, K. Mei, I. Ojalvo, J. Olsen, C. Palmer, P. Piroué, J. Salfeld-Nebgen, D. Stickland, C. Tully

University of Puerto Rico, Mayaguez, U.S.A.

S. Malik, S. Norberg

Purdue University, West Lafayette, U.S.A.

A. Barker, V.E. Barnes, S. Das, L. Gutay, M. Jones, A.W. Jung, A. Khatiwada, B. Mahakud, D.H. Miller, N. Neumeister, C.C. Peng, H. Qiu, J.F. Schulte, J. Sun, F. Wang, R. Xiao, W. Xie

Purdue University Northwest, Hammond, U.S.A.

T. Cheng, J. Dolen, N. Parashar

Rice University, Houston, U.S.A.

Z. Chen, K.M. Ecklund, S. Freed, F.J.M. Geurts, M. Kilpatrick, W. Li, B.P. Padley, J. Roberts, J. Rorie, W. Shi, Z. Tu, J. Zabel, A. Zhang

University of Rochester, Rochester, U.S.A.

A. Bodek, P. de Barbaro, R. Demina, Y.t. Duh, J.L. Dulemba, C. Fallon, T. Ferbel, M. Galanti, A. Garcia-Bellido, J. Han, O. Hindrichs, A. Khukhunaishvili, K.H. Lo, P. Tan, R. Taus

Rutgers, The State University of New Jersey, Piscataway, U.S.A.

A. Agapitos, J.P. Chou, Y. Gershtein, T.A. Gómez Espinosa, E. Halkiadakis, M. Heindl, E. Hughes, S. Kaplan, R. Kunnawalkam Elayavalli, S. Kyriacou, A. Lath, R. Montalvo, K. Nash, M. Osherson, H. Saka, S. Salur, S. Schnetzer, D. Sheffield, S. Somalwar, R. Stone, S. Thomas, P. Thomassen, M. Walker

University of Tennessee, Knoxville, U.S.A.

A.G. Delannoy, J. Heideman, G. Riley, S. Spanier, K. Thapa

Texas A\&M University, College Station, U.S.A.

O. Bouhali ${ }^{71}$, A. Celik, M. Dalchenko, M. De Mattia, A. Delgado, S. Dildick, R. Eusebi, J. Gilmore, T. Huang, T. Kamon ${ }^{72}$, S. Luo, R. Mueller, A. Perloff, L. Perniè, D. Rathjens, A. Safonov

\section{Texas Tech University, Lubbock, U.S.A.}

N. Akchurin, J. Damgov, F. De Guio, P.R. Dudero, S. Kunori, K. Lamichhane, S.W. Lee, T. Mengke, S. Muthumuni, T. Peltola, S. Undleeb, I. Volobouev, Z. Wang

Vanderbilt University, Nashville, U.S.A.

S. Greene, A. Gurrola, R. Janjam, W. Johns, C. Maguire, A. Melo, H. Ni, K. Padeken, J.D. Ruiz Alvarez, P. Sheldon, S. Tuo, J. Velkovska, M. Verweij, Q. Xu 


\section{University of Virginia, Charlottesville, U.S.A.}

M.W. Arenton, P. Barria, B. Cox, R. Hirosky, M. Joyce, A. Ledovskoy, H. Li, C. Neu, T. Sinthuprasith, Y. Wang, E. Wolfe, F. Xia

\section{Wayne State University, Detroit, U.S.A.}

R. Harr, P.E. Karchin, N. Poudyal, J. Sturdy, P. Thapa, S. Zaleski

\section{University of Wisconsin - Madison, Madison, WI, U.S.A.}

M. Brodski, J. Buchanan, C. Caillol, D. Carlsmith, S. Dasu, L. Dodd, B. Gomber, M. Grothe, M. Herndon, A. Hervé, U. Hussain, P. Klabbers, A. Lanaro, K. Long, R. Loveless, T. Ruggles, A. Savin, N. Smith, W.H. Smith, N. Woods

\section{t: Deceased}

1: Also at Vienna University of Technology, Vienna, Austria

2: Also at IRFU, CEA, Université Paris-Saclay, Gif-sur-Yvette, France

3: Also at Universidade Estadual de Campinas, Campinas, Brazil

4: Also at Federal University of Rio Grande do Sul, Porto Alegre, Brazil

5: Also at Université Libre de Bruxelles, Bruxelles, Belgium

6: Also at University of Chinese Academy of Sciences, Beijing, China

7: Also at Institute for Theoretical and Experimental Physics, Moscow, Russia

8: Also at Joint Institute for Nuclear Research, Dubna, Russia

9: Also at Helwan University, Cairo, Egypt

10: Now at Zewail City of Science and Technology, Zewail, Egypt

11: Also at Department of Physics, King Abdulaziz University, Jeddah, Saudi Arabia

12: Also at Université de Haute Alsace, Mulhouse, France

13: Also at Skobeltsyn Institute of Nuclear Physics, Lomonosov Moscow State University, Moscow, Russia

14: Also at CERN, European Organization for Nuclear Research, Geneva, Switzerland

15: Also at RWTH Aachen University, III. Physikalisches Institut A, Aachen, Germany

16: Also at University of Hamburg, Hamburg, Germany

17: Also at Brandenburg University of Technology, Cottbus, Germany

18: Also at MTA-ELTE Lendület CMS Particle and Nuclear Physics Group, Eötvös Loránd University, Budapest, Hungary

19: Also at Institute of Nuclear Research ATOMKI, Debrecen, Hungary

20: Also at Institute of Physics, University of Debrecen, Debrecen, Hungary

21: Also at Indian Institute of Technology Bhubaneswar, Bhubaneswar, India

22: Also at Institute of Physics, Bhubaneswar, India

23: Also at Shoolini University, Solan, India

24: Also at University of Visva-Bharati, Santiniketan, India

25: Also at Isfahan University of Technology, Isfahan, Iran

26: Also at Plasma Physics Research Center, Science and Research Branch, Islamic Azad University, Tehran, Iran

27: Also at Università degli Studi di Siena, Siena, Italy

28: Also at Kyunghee University, Seoul, Korea

29: Also at International Islamic University of Malaysia, Kuala Lumpur, Malaysia

30: Also at Malaysian Nuclear Agency, MOSTI, Kajang, Malaysia

31: Also at Consejo Nacional de Ciencia y Tecnología, Mexico city, Mexico

32: Also at Warsaw University of Technology, Institute of Electronic Systems, Warsaw, Poland 
33: Also at Institute for Nuclear Research, Moscow, Russia

34: Now at National Research Nuclear University 'Moscow Engineering Physics Institute' (MEPhI), Moscow, Russia

35: Also at St. Petersburg State Polytechnical University, St. Petersburg, Russia

36: Also at University of Florida, Gainesville, U.S.A.

37: Also at P.N. Lebedev Physical Institute, Moscow, Russia

38: Also at California Institute of Technology, Pasadena, U.S.A.

39: Also at Budker Institute of Nuclear Physics, Novosibirsk, Russia

40: Also at Faculty of Physics, University of Belgrade, Belgrade, Serbia

41: Also at INFN Sezione di Pavia ${ }^{a}$, Università di Pavia ${ }^{b}$, Pavia, Italy

42: Also at University of Belgrade, Faculty of Physics and Vinca Institute of Nuclear Sciences, Belgrade, Serbia

43: Also at Scuola Normale e Sezione dell'INFN, Pisa, Italy

44: Also at National and Kapodistrian University of Athens, Athens, Greece

45: Also at Riga Technical University, Riga, Latvia

46: Also at Universität Zürich, Zurich, Switzerland

47: Also at Stefan Meyer Institute for Subatomic Physics (SMI), Vienna, Austria

48: Also at Gaziosmanpasa University, Tokat, Turkey

49: Also at Adiyaman University, Adiyaman, Turkey

50: Also at Istanbul Aydin University, Istanbul, Turkey

51: Also at Mersin University, Mersin, Turkey

52: Also at Piri Reis University, Istanbul, Turkey

53: Also at Ozyegin University, Istanbul, Turkey

54: Also at Izmir Institute of Technology, Izmir, Turkey

55: Also at Marmara University, Istanbul, Turkey

56: Also at Kafkas University, Kars, Turkey

57: Also at Istanbul University, Faculty of Science, Istanbul, Turkey

58: Also at Istanbul Bilgi University, Istanbul, Turkey

59: Also at Hacettepe University, Ankara, Turkey

60: Also at Rutherford Appleton Laboratory, Didcot, United Kingdom

61: Also at School of Physics and Astronomy, University of Southampton, Southampton, United Kingdom

62: Also at Monash University, Faculty of Science, Clayton, Australia

63: Also at Bethel University, St. Paul, U.S.A.

64: Also at Karamanoğlu Mehmetbey University, Karaman, Turkey

65: Also at Utah Valley University, Orem, U.S.A.

66: Also at Purdue University, West Lafayette, U.S.A.

67: Also at Beykent University, Istanbul, Turkey

68: Also at Bingol University, Bingol, Turkey

69: Also at Sinop University, Sinop, Turkey

70: Also at Mimar Sinan University, Istanbul, Istanbul, Turkey

71: Also at Texas A\&M University at Qatar, Doha, Qatar

72: Also at Kyungpook National University, Daegu, Korea 